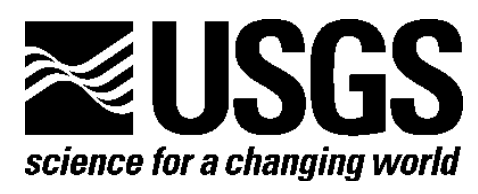

\title{
Quality Assurance and Quality Control of Geochemical Data: A Primer for the Research Scientist
}

By Nicholas J. Geboy and Mark A. Engle

Open-File Report 2011-1187

U.S. Department of the Interior

U.S. Geological Survey 


\section{U.S. Department of the Interior \\ KEN SALAZAR, Secretary}

\section{U.S. Geological Survey \\ Marcia K. McNutt, Director}

U.S. Geological Survey, Reston, Virginia 2011

For product and ordering information:

World Wide Web: http://www.usgs.gov/pubprod

Telephone: 1-888-ASK-USGS

For more information on the USGS-the Federal source for science about the Earth,

its natural and living resources, natural hazards, and the environment:

World Wide Web: http://www.usgs.gov

Telephone: 1-888-ASK-USGS

Suggested citation:

Geboy, N.J., and Engle, M.A., 2011, Quality assurance and quality control of geochemical data-A primer for the research scientist: U.S. Geological Survey Open-File Report 2011-1187, 28 p.

Any use of trade, product, or firm names is for descriptive purposes only and does not imply endorsement by the U.S. Government.

Although this report is in the public domain, permission must be secured from the individual copyright owners to reproduce any copyrighted material contained within this report. 


\section{Contents}

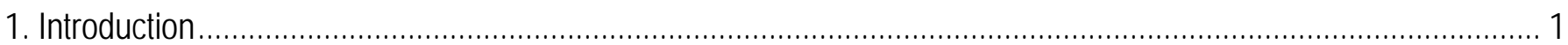

2. Considerations Prior to Sample Collection ....................................................................................... 1

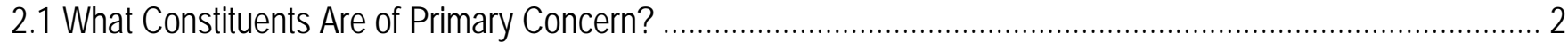

2.2 What Are the Proper Techniques and Laboratories to Determine the Constituents of Primary Interest? ............ 2

2.2.1 Common Interferences for Inorganic Elemental Analysis on ICP-MS................................................... 3

2.2.2 Common Interferences of Elemental Analysis by Emission and Absorption Spectrometry .......................... 3

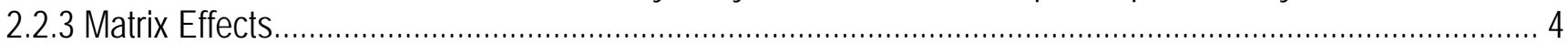

2.3 What Are the Geochemical Abilities of the U.S. Geological Survey Energy Resources Program Laboratories in Denver?

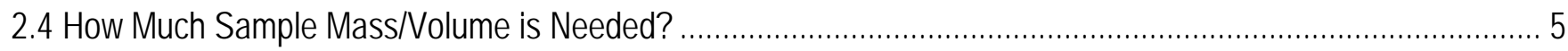

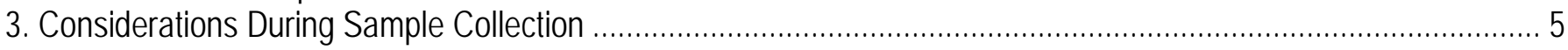

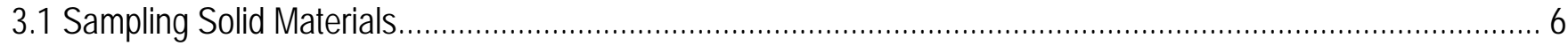

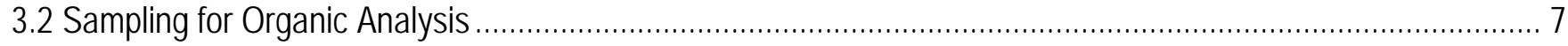

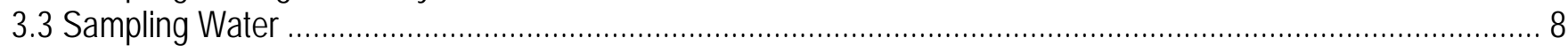

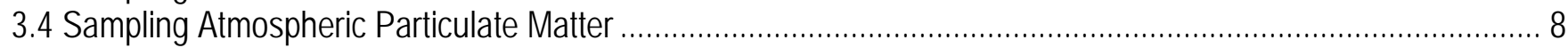

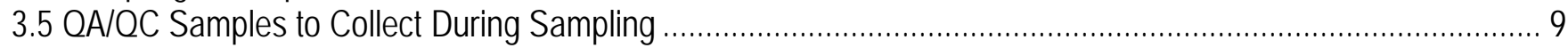

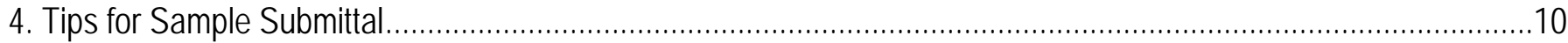

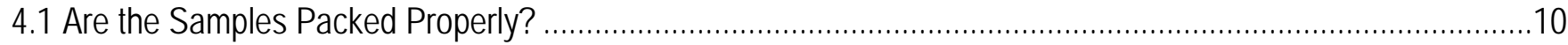

4.2 What Else Needs to Be Included With the Unknown Samples?

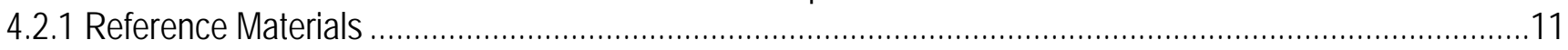

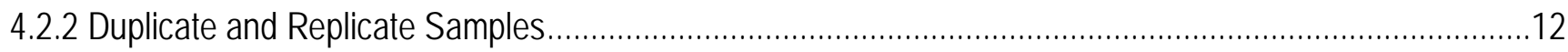

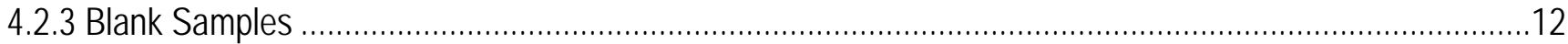

4.2.4 Suggested Inclusion of QA/QC Samples to Accompany a Batch of Samples Submitted to an Analytical

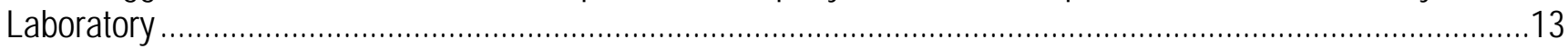

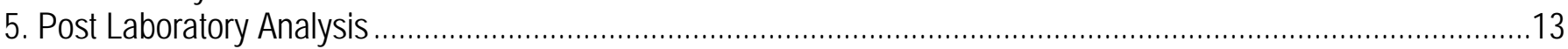

5.1 Using Field Blanks to Determine the Method Detection Limit.....................................................................

5.2 Examples of the Importance of Scrutinizing Data with Respect to QA/QC

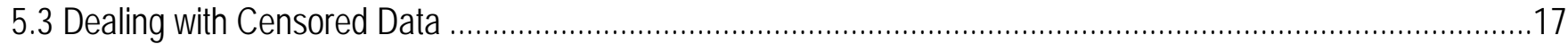

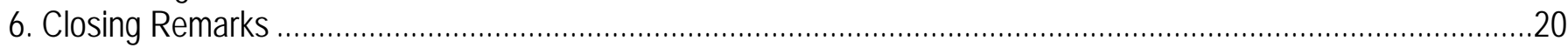

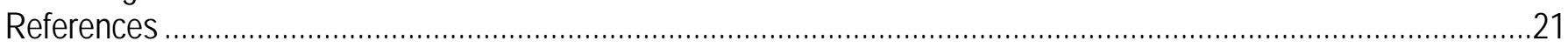

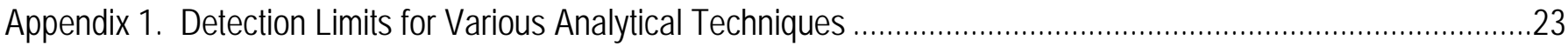

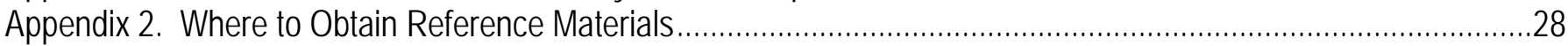

\section{Figures}

1. Data used to generate a rhenium $(\mathrm{Re})$-osmium $(\mathrm{Os})$ isochron ............................................................

2. Vanadium, rubidium, and strontium concentration data in atmospheric particulate matter collected from Woods Hole, Massachusetts, in February 2008 ……....................................................................

3. Leachate data from coal combustion products by two different analytical procedures, which do not display a

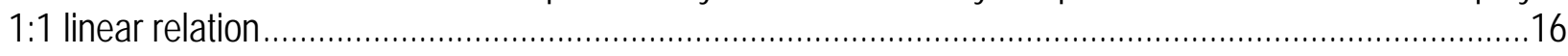

4. Leachate data from coal combustion products by two different analytical procedures following a transposition of laboratory identifications . 


\section{Tables}

1. Sodium ( $\mathrm{Na}$ ) concentration data for atmospheric total suspended particulate matter from Woods Hole, Massachusetts, with summary statistics.

2. Comparison of summary statistics for the dataset when arbitrary detection limits are set and four different nondetect statistical methods are employed.

\section{Appendix Figures}

A1. Instrument detection limits for inductively coupled plasma-atomic emission spectrometry (ICP-AES) .........23

A2. Instrument detection limits for inductively coupled plasma-mass spectrometry (ICP-MS) ..........................24

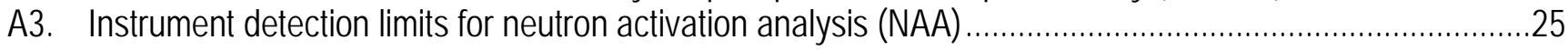

A4. Instrument detection limits for X-ray fluorescence spectrometry (XRF) ...............................................26

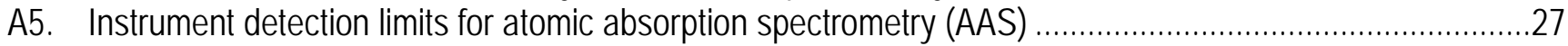




\section{Conversion Factors}

Inch/Pound to SI

\begin{tabular}{rll}
\hline Multiply & By & To obtain \\
\hline & Mass & \\
\hline pound, avoirdupois (lb) & 0.4536 & kilogram (kg) \\
\hline
\end{tabular}

SI to Inch/Pound

\begin{tabular}{|c|c|c|}
\hline Multiply & By & To obtain \\
\hline \multicolumn{3}{|c|}{ Length } \\
\hline meter (m) & 3.281 & foot $(\mathrm{ft})$ \\
\hline \multicolumn{3}{|c|}{ Mass } \\
\hline gram (g) & 0.03527 & ounce, avoirdupois (oz) \\
\hline
\end{tabular}

Temperature in degrees Celsius $\left({ }^{\circ} \mathrm{C}\right)$ may be converted to degrees Fahrenheit $\left({ }^{\circ} \mathrm{F}\right)$ as follows: ${ }^{\circ} \mathrm{F}=\left(1.8 x^{\circ} \mathrm{C}\right)+32$ 


\title{
Quality Assurance and Quality Control of Geochemical Data: A Primer for the Research Scientist
}

\author{
By Nicholas J. Geboy and Mark A. Engle
}

\section{Introduction}

Geochemistry is a constantly expanding science. More and more, scientists are employing geochemical tools to help answer questions about the Earth and earth system processes. Scientists may assume that the responsibility of examining and assessing the quality of the geochemical data they generate is not theirs but rather that of the analytical laboratories to which their samples have been submitted. This assumption may be partially based on knowledge about internal and external quality assurance and quality control (QA/QC) programs in which analytical laboratories typically participate. Or there may be a perceived lack of time or resources to adequately examine data quality. Regardless of the reason, the lack of QA/QC protocols can lead to the generation and publication of erroneous data. Because the interpretations drawn from the data are primary products to U.S. Geological Survey (USGS) stakeholders, the consequences of publishing erroneous results can be significant. The principal investigator of a scientific study ultimately is responsible for the quality and interpretation of the project's findings, and thus must also play a role in the understanding, implementation, and presentation of QA/QC information about the data.

Although occasionally ignored, QA/QC protocols apply not only to procedures in the laboratory but also in the initial planning of a research study and throughout the life of the project. Many of the tenets of developing a sound QA/QC program or protocols also parallel the core concepts of developing a good study: What is the main objective of the study? Will the methods selected provide data of enough resolution to answer the hypothesis? How should samples be collected? Are there known or unknown artifacts or contamination sources in the sampling and analysis methods? Assessing data quality requires communication between the scientists responsible for designing the study and those collecting samples, analyzing samples, treating data, and interpreting results.

This primer has been developed to provide basic information and guidance about developing QA/QC protocols for geochemical studies. It is not intended to be a comprehensive guide but rather an introduction to key concepts tied to a list of relevant references for further reading. The guidelines are presented in stepwise order beginning with presampling considerations and continuing through final data interpretation. The goal of this primer is to outline basic QA/QC practices that scientists can use before, during, and after chemical analysis to ensure the validity of the data they collect with the goal of providing defendable results and conclusions.

\section{Considerations Prior to Sample Collection}

Gathering meaningful data begins long before the laboratory analyst ever receives the samples. In the initial stages of any scientific study, a fundamental scientific question is posed along with a conceptual scheme of an acceptable approach to answer the research question. One piece of this scheme 
should include developing (even if informally) a sampling plan, which is a fundamental step in collecting relevant data (Luppens and others, 1992; Ramsey, 1997). When developing a sampling plan, it is important to keep the aims of the study in mind and to determine what constitutes a representative sample of the material being investigated. Questions to keep in mind include: How many samples are required to answer the scientific question posed? Do these samples need to meet some sort of temporal or spatial resolution? Do the samples need to be representative of something larger than themselves? What is the minimum mass or volume of each sample needed to perform all necessary analyses, keeping in mind reserve splits may be necessary? What is the minimum number of samples required for statistical significance? What sorts of field QA/QC samples (blanks, duplicates, and so forth) are appropriate? Some of these questions may be answered with the assistance of a literature search. Often other researchers have published information on proper sampling methods, preservation methods, potential pitfalls, and analytical techniques on similar topics or study areas. Field time is expensive, and developing a thoughtful sampling plan prior to collecting samples gives a researcher more time in the field to actually be sampling and ensures that the samples that were collected will appropriately address the scientific objectives of the study.

\subsection{What Constituents Are of Primary Concern?}

Just because an analytical test can be performed does not mean it has to or should be performed. Identifying which elements or compounds are the most vital to answer the questions posed will streamline a study and make it more insightful. For example, if determining the major oxide concentrations within a suite of coals is all that is required to answer a scientific question, then performing a blanket analysis for the entire periodic table is unnecessary and may come at the expense of the required analyses. Practically speaking, sampling procedures and contamination sources vary widely depending on the analytes of interest. Selectively choosing which analyses best address the objectives of a study allows the analyst to home in on more precise data, because the key components to be tested will determine the best method to use. If it is ever unclear which constituents would best help answer a scientific question, searching the literature to see what has or has not worked for past researchers is a useful start.

\subsection{What Are the Proper Techniques and Laboratories to Determine the Constituents of Primary Interest?}

Any given constituent can be measured by using a variety of analytical techniques, both qualitative and quantitative. Qualitative techniques include electron probe microanalyzer (EPMA), X-ray diffraction (XRD), nuclear magnetic resonance (NMR), infrared spectroscopy (IR), and so forth. Quantitative techniques include inductively coupled plasma-mass spectrometry (ICP-MS), inductively coupled plasma-atomic emission spectrometry (ICP-AES), thermal ionization mass spectrometry TIMS), gas chromatography-mass spectrometry (GC-MS), X-ray fluorescence (XRF), neutron activation analysis (NAA), EPMA, and quantitative XRD, among others. These methods have varying detection limits, interferences, restrictions, and costs, and these factors may make data from specific methods unsuitable to provide the requisite precision or accuracy needed to answer the question at hand. To assist in selecting an appropriate method, detection limits for a variety of analytical methods can be found in appendix 1.

As an example of where certain analytical methods are not appropriate for certain samples, consider a study involving measuring $\mathrm{Hg}$ in flue gas. Concentrations average 1,500 parts per million by volume for sulfur dioxide gas $\left[\mathrm{SO}_{2}(\mathrm{~g})\right]$ in flue gas emissions. Some commercial types of mercury 
analyzers exhibit a strong interference in the presence of $\mathrm{SO}_{2}(\mathrm{~g})$, so research must be done using only an instrument in which there is no interference or one which corrects for the interference.

\subsubsection{Common Interferences for Inorganic Elemental Analysis on ICP-MS}

Mass spectrometers, which represent the measurement portion of an ICP-MS, separate isotopes on the basis of their mass-to-charge ratio $(\mathrm{m} / \mathrm{e})$. Isobars are nuclides with the same number of nucleons and isobaric interferences occur in mass spectrometry when isotopes from different elements of equal $\mathrm{m} / \mathrm{e}$ are present in significant concentrations in the same sample solution. Often, when an element isobarically interferes with another, a different isotope of the analyte element must be selected. For example, the most abundant isotope for calcium is ${ }^{40} \mathrm{Ca}$. ICP-MS utilizes an argon torch, and 99.6 percent of argon has a mass of 40 atomic mass units (amu). Therefore, they cannot be distinguished from one another by a magnetic mass analyzer. Analysts can get around this problem by analyzing for the second most abundant isotope of calcium, ${ }^{44} \mathrm{Ca}$. Strontium, however, readily substitutes for calcium, so both are commonly found in the same sample solution. The most abundant isotope of strontium has a mass of 88, and ${ }^{88} \mathrm{Sr}^{2+}$ has the same m/e as ${ }^{44} \mathrm{Ca}^{+}$, so a magnetic analyzer on an ICP-MS cannot distinguish between them either. The ${ }^{43} \mathrm{Ca}^{+}$ion, however, has no common interferences and, therefore, would be a better isotope to determine the concentration of calcium within a sample. Although the scientist submitting samples has little control over which isotopes the analyst uses, communicating the elements of interest and basic information about the matrix of the samples may help the analyst make a more informed decision.

Isobaric interferences are a concern mainly when monoisotopic elements are involved. The monoisotopic elements are ${ }^{9} \mathrm{Be},{ }^{23} \mathrm{Na},{ }^{27} \mathrm{Al},{ }^{45} \mathrm{Sc},{ }^{55} \mathrm{Mn},{ }^{75} \mathrm{As},{ }^{89} \mathrm{Y},{ }^{103} \mathrm{Rh},{ }^{127} \mathrm{I},{ }^{133} \mathrm{Cs},{ }^{141} \mathrm{Pr},{ }^{159} \mathrm{~Tb},{ }^{165} \mathrm{Ho}$, ${ }^{169} \mathrm{Tm},{ }^{197} \mathrm{Au}$, and ${ }^{232} \mathrm{Th}$; no singly charged isotopes overlap with any of them. However, ${ }^{206} \mathrm{~Pb}^{2+} \mathrm{can}$ interfere with ${ }^{103} \mathrm{Rh}^{-}$, and some molecules formed in the plasma also can interfere ${ }^{40} \mathrm{Ar}^{35} \mathrm{Cl}^{-}$overlaps with ${ }^{75} \mathrm{As}^{-}$). If the scientist is confident there is no measurable chlorine in the sample, this interference with arsenic can be avoided by not using $\mathrm{HCl}$ in sample digestion or preservation. Although the analyst may be aware of these problems and know how to work around them, it is prudent for the scientist to also be aware of such issues and communicate with laboratory personnel to help avoid potentially interference-laden results.

\subsubsection{Common Interferences of Elemental Analysis by Emission and Absorption Spectrometry}

Emission spectroscopy refers to analytical techniques that excite the atoms in a sample and determine concentrations by measuring the characteristic wavelengths emitted when the atoms relax. These techniques include ICP-AES, which excites the atoms with heat, and an electron probe, which excites the atoms with electron bombardment. There are literally thousands of spectral interferences in emission spectroscopy. Most laboratories, however, are prepared for this interference and measure for characteristic lines that do not overlap significantly with other common elements. For ICP-AES, if there is any question about the reliability of a particular instrument or laboratory, the most recent spectral interference study results should be requested. These studies, performed annually, ensure there are no direct or wing overlaps or near neighbors that affect the background around specific analytes (elements of interest).

Absorption spectroscopy is similar to emission spectroscopy. Like in emission spectroscopy, samples are excited to higher energy levels. As the name implies, however, characteristic wavelengths of the energy absorbed, rather than emitted, are measured and used to determine concentration. Atomic absorption spectroscopy (AAS) is one such technique and is used in the analysis of mercury in solid materials. Spectral interferences may also occur in AAS, but perhaps more important concerns are 
chemical interferences. Chemical interferences occur when elements being analyzed form molecules that are not fully atomized by the flame and thus do not absorb the characteristic wavelength of energy. Common examples of chemical interferences occur when phosphate is present in a sample where $\mathrm{Mg}$, $\mathrm{Ca}$, or $\mathrm{Ba}$ concentrations are being determined or when silica is present in a sample where Mn is being analyzed. Other interferences and suggestions for overcoming them can be found in U.S. Environmental Protection Agency (2007).

Although it is not necessary for all researchers to be fluent in the workings of ICP-AES, EPMA, or AAS, these analytical methods are commonly used by geoscientists, and thus it is helpful to have a basic understanding of them. Before submitting samples to a laboratory, it is important to consider what components need to be analyzed and what potential interferences are associated with each technique. Discussing standard operating procedures with the laboratory analyst or manager is a simple way to resolve any doubts about a method, determine the best way to proceed with a project, and ensure that both parties are on the same page with respect to what is in the samples and what could potentially go awry during the analysis.

\subsubsection{Matrix Effects}

A common group of interferences present in all of the above mentioned analytical techniques is referred to as matrix effects. The matrix, defined as all of the components of a sample other than the analyte, can affect the quality of the results obtained from the geochemical analysis if the sensitivity of the analyte is dependent on any component of the matrix. Chemical effects are an example of matrix effects. Another example of matrix effects occurs when using a plasma source (for example, ICP-MS and ICP-AES). High concentrations of total dissolved solids in a sample can result in deposits accruing on the orifice of the sampling cone, which leads to signal drift over small time scales and loss of precision.

Several protocols can be used for detecting and compensating for matrix effects. If extra sample is available for the analysis, a split can be taken from one or more samples and spiked with a known concentration of the analytes of interest prior to sample preparation and analysis. Analytical results from these splits, known as matrix spikes, are compared to the analysis of the original samples to determine how much of the spiked material was recovered. If the recovery of the analyte is outside the accepted limits set by that laboratory or study, then there are significant matrix effects, and the data for that analyte are considered to be questionable and further discussion with the laboratory may be required about possible methods to circumvent the problem. Even if the recovery rates fall within the acceptable limits, it does not prove an absence of matrix effects. Rather, it suggests that if matrix effects are present, they are no larger than those typically present for a variety of matrices that underwent the same preparatory and analytical procedures. Discrepancies can be attributed to matrix effects only if other QA/QC samples are falling within their accepted ranges and the concentration of the spike is much higher than the ambient analyte. If significant matrix effects are associated with the analysis, the effects may be overcome by ensuring the samples and calibration standards have the same matrix. This technique is called matrix matching. With matrix matching, the calibration curve for the instrument has already taken into account all matrix effects and essentially corrected for them. It is important, therefore, to inform the analyst of the nature of the samples submitted and to include reference materials with similar matrices. In some cases, a lack of clear communication with a laboratory about the nature of the samples can lead to unintentional matrix problems. While properly designed matrix spikes can be one of the best methods to identify potential problems with the analysis (as they include information on both the calibration and interferences), not all laboratories routinely run them and therefore their inclusion 
may be an important point of discussion with the laboratory prior to submitting the samples. More detailed information on matrix spikes can be found in U.S. Army Corps of Engineers (2005).

Once the proper analytical method is identified and possible analytical interferences are considered, a laboratory must be chosen to perform that method. Many laboratories around the country can be used for common analyses. If the component of interest is more specialized, however, an appropriate laboratory must be identified. For example, the analysis for mercury isotopes would most likely require selection of a research laboratory employing a highly specialized analyst because of the complexity of the method.

\subsection{What Are the Geochemical Abilities of the U.S. Geological Survey Energy Resources Program Laboratories in Denver?}

The USGS Energy Resources Program (ERP) laboratories in Denver, Colorado, have established standard operating procedures (SOPs) for the following methodologies, presented in no particular order: 525 degrees Celsius $\left({ }^{\circ} \mathrm{C}\right.$ ) and $750{ }^{\circ} \mathrm{C}$ ashing of coal; American Petroleum Institute (API) gravity; bitumen extraction by Soxhlet; fractionation of oil or bitumen extract by column chromatography; elemental analyzer determination for carbon, hydrogen, nitrogen, oxygen, and sulfur; gas chromatography (GC); gas quantification; GC of aromatic hydrocarbons; GC of saturate hydrocarbons; GCMS analysis of biomarkers; loss on ignition; low temperature coal ashing; microwave digestion; maltene preparation; direct mercury analysis in coal, rock, sediment, and soil by using a DMA-80; moisture in coal; trace element analysis by ICP-MS (Elan 6100); Optima isotope ratio mass spectrometer coupled with elemental analyzer for stable isotopes analysis on solid and liquids; PANalytical X-ray diffractometer; sample preparation for analysis by organic petrology; selenium analysis and digestion; sulfur analysis on a LECO-SC632; and trace digestion. Additional and updated information on the ERP laboratories (including instrument models) can be found on their Web page at http://energy.cr.usgs.gov/gg/geochemlab/index.html.

\subsection{How Much Sample Mass/Volume is Needed?}

Collecting the appropriate mass or volume of sample is important not only to lower uncertainty but also to ensure there is enough sample to complete all desired analyses and QA/QC checks and keep a reserve split. As an example, for coal, ERP laboratories in Denver suggest 50 grams (g) of sample for major and trace elemental analysis. Some commercial laboratories suggest about 5 to $10 \mathrm{~g}$ to complete ultimate/proximate, $\mathrm{F} / \mathrm{Cl}$ and loss on ignition (LOI) analyses. Further, 1 to $3 \mathrm{~g}$ are needed to make a pellet for organic petrography or microprobe work. Therefore, if coal or coal combustion products (CCPs) are being sampled, a minimum of $60 \mathrm{~g}$ is needed for total analysis. Keep in mind, however, that sample may be lost during grinding and other preparatory work, so it would be best to collect at least 100 g. Additional sample may also be necessary for matrix spikes and reserve splits. Each study and medium are different, so research into analytical methodology is essential before any sampling event.

\section{Considerations During Sample Collection}

Sampling involves collecting both actual samples, which will help answer scientific questions, and QA/QC samples, which provide quantitative information on the quality of the analytical results. QA/QC samples, such as blanks and duplicates, can only add to the robustness of a study and are critical even despite a potential perceived lack of time and (or) money to collect and analyze them. They are important insofar as they can be used to account for any outside influences and offer insight into potential analytical problems. The usefulness of QA/QC samples depends on how honestly they are 
collected and treated. It may be tempting to treat QA/QC samples with extra care to, for example, make a blank contribution appear as low as possible. If an actual sample, however, is not treated with the same care, then the blank sample will not accurately reflect the reality of the sample. Each of the QA/QC samples described below should be treated like any other sample in terms of collection, storage, preservation, cleaning, labeling, shipment, and any other processes involved.

Contamination of sample is not restricted to laboratory error. Understanding possible QA/QC concerns during sampling can prevent inadvertently degrading a sample prior to returning from the field. For instance, in a study on the environmental effects of coal, the focus may be on arsenic, selenium, mercury, and polycyclic aromatic hydrocarbons (PAHs). Each of these constituents exhibits its own sampling and analytical challenges; however, the inorganic constituents will require much different sampling and preservation methods than the PAHs. If samples are collected using only methods for PAHs, they may not be suitable for further analysis of the three trace elements. In the same way, it could be a waste of resources to collect samples for measurement of elevated sodium, a major cation in water that can cause damage to soils in arid environments, using methods designed for lowlevel trace concentrations (for example, using a particle-free sampling suit, arm-length gloves, doublebagged Telfon ${ }^{\mathrm{TM}}$ sample bottles, and so forth). Regardless of the media and nature of the samples being collected, recognized standards should be reviewed, and method sections of recent papers may be checked to ensure established sampling protocols are being followed.

General points to consider when sampling different media are given below. By no means are the following sections complete but rather offer ideas to consider when sampling. Some of the proper standards for sampling various media are identified.

\subsection{Sampling Solid Materials}

For USGS ERP scientists, solid geomaterials are often synonymous with coal, CCPs, and, occasionally, overburden. Sampling coal for geochemical analysis can be done both in the field and onsite at a powerplant or core storage facility. In any case, coal samples can be stored in plastic bags within metal tins to minimize moisture loss, coal oxidation, and cross contamination among the samples. For temporary storage, plastic bags without the tins are acceptable; however, some researchers have noted that over a period of years plastic bags deteriorate, leaving the samples exposed to each other and the atmosphere. Metadata must be obtained from the powerplant/mine with respect to coal type and grade, source, sampling date, time, preservation, and so forth. Some level of metal contamination is impossible to avoid if the coal that has already been mined and transported is stored in direct contact with metals and other contaminants. The assumption is that the level of metal contamination is so far below the concentration of elements within the coal that it is negligible, but this may not always be true.

It is important to note weathering rinds and "nuggets" when preparing solid samples for analysis. Weathering rinds are not representative of the actual product that is being sampled but instead have exchanged components with the surrounding environment. Nugget effects occur when a component of the sample (such as a mineral grain) contains a disproportionate amount of a key element of interest or if it is heterogeneous on the scale of single sample volumes. For example, Illinois Basin coal may contain trace amounts of sphalerite, which can include cadmium. If the aliquot of coal sampled contains an unrepresentatively high amount of sphalerite grains, the measured cadmium concentration would be misleadingly high. Although it is impossible to avoid all nuggets and rinds, their inadvertent inclusion may explain anomalously high data points that should not be considered representative of the sample. However, if the study is related to coal quality, rinds and nuggets should be included in the analysis because mining operations do not separate them prior to coal combustion. 
Sampling coal cores presents some unique concerns worth addressing. It can be difficult to quantify how much core sample is needed to be truly representative. Each core should be evaluated individually in terms of lithology and composition. Distinct megascopic lithologies or facies should be sampled separately. In the case of coal assessments, inorganic partings are sampled separately, but not discarded, as their inclusion with the coal will increase ash content and lower BTU output (in case the coal is not preferentially mined around the partings). Coal cores may also be contaminated from drilling fluids. The exterior of cores should be cleaned prior to any chemical analysis. If possible, it may be preferable to use the interior of the core for more sensitive analyses such as trace elements. A comprehensive review of sampling coal core is available in Manual on Drilling, Sampling, and Analysis of Coal (Luppens and others, 1992).

Solid CCPs, including bed or bottom ash, fly ash, and flue gas desulfurization, among others, are also intrinsically contaminated to some degree. Nonetheless, it is important to store CCPs separately in sealable containers to minimize cross contamination. When splitting samples to achieve representative cuts, riffle splitting is the ASTM International standard (ASTM D2013). If low concentration isotopic analyses need to be done, however, it is better to roll, cone, and quarter the samples to prevent unnecessary contact with metal.

The USGS occasionally engages in overburden studies that involve sampling solid materials from core. Overburden is material that rests above minable materials (that is, coal) that is removed prior to surface mining and then put back in place during the reclamation process as outlined in the Surface Mining Control and Reclamation Act of 1977. As with coal core sampling, it is important to note lithologic changes within the core and sample in a way that is representative of those changes. That way if one lithology is found to contain higher concentrations of toxic elements than other lithologies, it can be filled in first during reclamation, minimizing the risk of it being aerially exposed, and thus mechanically weathered into the environment.

Appropriate procedures for sampling coal can be found in ASTM D7430-10b. In addition, ASTM D4596 (Coal Seam Face Channel Sampling) and D5192 (Coal Core Sampling) may be useful. Standards also are available from ASTM Committee D18 (soils) that apply to overburden and soil sampling.

\subsection{Sampling for Organic Analysis}

Samples collected for organic analysis, such as surface waters, produced waters, and natural gas samples, are easily contaminated by oils, plastics, sunscreen, and many other common items not of concern when sampling for inorganics. Because most traditional sampling techniques involve plastic gloves, plastic bags, labeling tape, pencils/markers, and so forth, extra precaution must be taken to obtain meaningful organic data.

Prior to being placed in sample bags, geologic samples should be wrapped in aluminum foil or other low organic carbon-containing material to prevent direct contact with the plastic. Aluminum foil is easily pretreated by combustion at $400{ }^{\circ} \mathrm{C}$ for 16 hours to oxidize any organic carbon present (Brocks and others, 2003). Nonetheless, many commercially available aluminum foils contain one oiled side. The sample should be wrapped in such a manner that it does not touch the oiled side of the foil. When labeling samples, it is best to mark the sample name on the foil rather than on the sample itself. Both inks and pencil "lead" are organic and may leave trace amounts of residue behind. If gloves are required, appropriate gloves, such as nitrile, are recommended.

Once collected, samples being prepared for organic analysis must be treated with caution. When glass fiber is used as a collection substrate of organic compounds, it should be combusted at $400{ }^{\circ} \mathrm{C}$ for 16 hours or as indicated in appropriate methods. When solid samples are ground for organic analysis, 
the ring mill should be cleaned between samples by washing with water and grinding two or three times with quartz sand (which has been annealed at $900{ }^{\circ} \mathrm{C}$ for 24 hours) at 60 -second intervals (Brocks and others, 2003).

\subsection{Sampling Water}

ERP scientists collect water samples for a variety of reasons but especially when investigating the effects of energy resource use on the environment and human health. Although fresh and saline waters must be treated differently analytically, the QA/QC protocols during sampling are similar. Plastic, glass, or Teflon ${ }^{\mathrm{TM}}$ collection bottles should be precleaned with acid (if necessary) and triple rinsed with the water to be sampled prior to actual collection. It may be necessary to use opaque or amber bottles to prevent photochemical reactions from occurring, which could change the concentrations of vulnerable components. The amount of headspace may also be an issue for more volatile elements. If suspended particles are not of interest, it is prudent to pass the water sample through a 0.45 micrometer $(\mu \mathrm{m})$ or finer filter during collection to prevent dissolved material from adsorbing onto the larger particles, which can be removed from the solution. To preserve the sample between collection and analysis, it is standard practice to acidify solutions for later elemental analysis with 0.1 molar (M) nitric acid (high purity, if necessary), unless the sample will be analyzed for anions or titrated to determine alkalinity, as the nitric acid will minimize solute deposition on the container walls. Hydrochloric acid should only be used if chlorine is not of interest to the research, while still considering the ArCl interference on ICP-MS and ICP-AES discussed in section 2.2.1. Promptly refrigerating or placing samples on ice will slow biological activity, which may regulate trace metal concentrations such as Ni, V, Fe, and Mo. Saline waters from the sea, estuaries, or deep boreholes may have lower stability and, therefore, require more rapid preservation through acidification and (or) refrigeration.

If water samples are being collected for organic analysis, nitrile gloves should be worn. As with inorganic analysis collection, samples should be collected in amber or opaque bottles, filtered, and refrigerated. Samples may require preservation with dichloromethane or other antibacterial additive to prevent bacterial growth within the filtered sample. The scientist must be aware of situations that could affect the results, such as seasonal, biological, and meteorological events, which may prevent a sample from being representative or "background," and many samples may be needed for both temporal and spatial comparisons. For more information on sampling water, the USGS has produced the National Field Manual for the Collection of Water-Quality Data (U.S. Geological Survey, 2006).

\subsection{Sampling Atmospheric Particulate Matter}

Collecting particulate matter from the atmosphere requires pumping a known volume across impactors, which segregate particles on the basis of aerodynamic diameter, or through filters. Several different instruments can be used to collect particulate matter, each with its own characteristic particle size range and flow rate range. Flow rate through the samplers and duration of collection must be calculated prior to arrival in the field and adjusted onsite to ensure enough sample is amassed for chemical analysis. In addition, because filters are made of different media, their use depends on the nature of the compounds of interest. Commonly, cellulose and Teflon ${ }^{\mathrm{TM}}$ filters are used when sampling for trace metals, prefired quartz fiber is used when sampling for $\mathrm{Hg}$, and prefired glass fiber is used when sampling for organics.

Impactors and stages can be easily contaminated by atmospheric particulate matter prior to the start of collection. For studies focusing on trace elements or constituents that are prone to contamination, special care should be taken. Vinyl gloves and ultra-low particle (for example, Tyvek ${ }^{\circledR}$ ) 
lab coats, which are approved for use in clean room settings, should minimize contamination of the filter during loading of filters into sample holders and stages; ideally, this process is done within a laminar flow hood. Transportation of loaded stages in multiple layers of bags or other containers prior to sample collection will also help minimize potential contamination. The physical sampler should also be wiped with a low-particle cloth immediately before the stages are put in place. The particular gloves, containers, and cleaning materials and solutions should be appropriate for the analytes of interest so they do not readily contaminate the sampling media. Ideally, the samplers should be positioned at least 2 meters above the ground to ensure that a representative sample of the lower atmosphere is being collected that is not overly biased by large, saltating particles. Following collection, filters should be split (if necessary and with precleaned ceramic or metal scissors, depending on the compounds of interest) and stored in a freezer (if volatile compounds are being investigated) for preservation.

Many different methods can be used for sampling atmospheric particulate matter. A list of the various U.S. Environmental Protection Agency (EPA) standards is available (U.S. Environmental Protection Agency, 1999).

\subsection{QA/QC Samples to Collect During Sampling}

- Field Blanks - A field blank is a sample or sampling media (for example, filter) that has been exposed to the same field and transport conditions as the samples and is used to distinguish ambient contamination, which is attributed to collection and transportation processes, from actual sample contribution. For example, if a water sample is collected from a well in Pennsylvania and then shipped to a laboratory, high purity water would be brought into the field, transferred to another collection vessel, and shipped to the laboratory with the aqueous sample from the well. Another example would be filters to collect particulate matter. Field blanks for the filters would be created by placing the media into a filter holder and placing the holder at the sampling site but never running air through the filter.

- Trip Blanks - These samples are nearly identical to field blanks except the samples or sampling media cases are never removed from their packaging. In the water example above, a trip blank consists of water brought from the laboratory to the field site but never transferred to a collection vessel. Comparing the trip blank to the field blank can help determine if contamination was contributed from the field environment or from the environment the samples are exposed to during transport.

- Duplicates and Replicates - This report defines duplicates as samples collected in tandem with one another from the same source at the same time in the field. Replicates, on the other hand, are defined as repeat analyses performed on different aliquots of the same sample. Replicates may either be field replicates or laboratory replicates depending on the location of the sample analysis. Specific types of duplicates are discussed in more detail below. Duplicates provide information on both the heterogeneity of the sampling material (can be used to identify "nugget” effects) as well as the analytical precision. Replicates should be more representative of analytical variability; comparison of the two can be useful in quantifying and determining the source of analytical variability. Poorer precision in duplicates relative to replicates may indicate a lack of sample homogenization, and comparable results may indicate that analytical variability controls precision.

- Site/Study Reference Sample - In the case of a long-term or multiple campaign study (or if a large number of samples require the set to be split up and submitted as smaller individual batches), it may make sense to collect an extremely large volume of a single sample. This sample can be homogenized and submitted to the laboratory with each batch of samples (discussed in section 4) and used to track analytical variation between or among batches. 
- Compound Loss Sample - Although field and trip blanks are important in identifying sources of contamination during sampling, there is also a potential to preferentially lose compounds from the sample during the sampling events. This is more important for highly volatile or short-lived compounds. Although rarely done, a previously collected sample or aliquot of a reference sample can be shipped to the study area and then brought back to the laboratory following the path of a trip blank. Comparison of pre- and post-sampling analyses of the sample can be used to provide information on possible compound loss and the efficacy of preservation techniques in field conditions.

- Equipment Blanks - Equipment blanks reveal contamination from any equipment used during sample collection or preparation, such as from a total suspended particulate air sampler or from a water sampling pump and tubing. These samples can be among the most important QA/QC samples because they provide important information on how little or how much the sampling and preparation methods affect the results of the samples. In some cases, equipment blanks can be compared directly against field and trip blanks to provide quantitative information on contamination from sampling and preparation equipment. For instance, using the example of well sampling again, high purity water brought from the laboratory and pulled through the same pump used to sample the well can be used to directly estimate contributions of specific compounds in concentration units as a result of using the tubing and pump. In other cases, however, equipment blanks can provide only qualitative information on contamination from sampling. In the case of soil sampling, an equipment blank can be collected by rinsing off the unused sampling trowel with high purity water and collecting the rinsate. Comparison of the rinsate with unused high purity water may be helpful in identifying compounds on the trowel, which were added to the soil sample, but no direct determination of how much compound per sample can be made.

- Cleaning Agent Blanks - If any agents are used to clean field or laboratory equipment, it may be worthwhile to have them analyzed, especially if trace work is being done. Cleaning agents may include water, anionic detergent, or commercial products such as surface cleaners. For example, if a product such as an all-purpose cleaner is used to clean a laminar flow hood under which samples are prepared for trace element analysis, it may be prudent to analyze the cleaner to make sure its residue is not likely to contaminate the samples. As with some of the equipment blanks, cleaning agent blanks are more typically qualitative but can provide important clues on the efficacy and appropriateness of cleaning methods. While cleaning agents are not the most likely source of contamination, they are worth investigating if blanks are higher than expected.

\section{Tips for Sample Submittal}

The preparation of samples for laboratory submission includes proper packaging and providing appropriate QA/QC samples in the suite. Addressing the following questions when submitting samples can help ensure they do not become contaminated en route and that there is a sufficient accompaniment of QA/QC samples.

\subsection{Are the Samples Packed Properly?}

When samples are packed for shipment to the analytical laboratory, the samples should be stored in a way that will prevent them from having direct physical contact with each other, ideally in air tight containers. Ice packs should be used if the samples need to remain chilled during transport (such as organics or other volatile constituents), as the cold temperature slows oxidation and loss of some elements and inhibits biological activity. If the samples do need to remain chilled, they should be 
shipped at the beginning of the week to prevent a weekend arrival when the package may be left to sit on a warm loading dock for a day or two. Likewise, some solid samples should be stored in ways that minimize exposure to extreme hot or cold temperatures. Contact the laboratory to make sure that someone will be available to process the samples when they arrive. In addition, it is important to ship samples by a method that allows for tracking of packages so that misdirected parcels can be located. In some studies, background samples are collected in addition to potentially anomalous or contaminated samples. In this case, it may be helpful to ship these two groups separately to prevent contamination of the control group, especially by volatile compounds.

\subsection{What Else Needs to Be Included With the Unknown Samples?}

Several key pieces of information can be gleaned from various types of QA/QC samples submitted to and provided by the laboratory. The following sections discuss how reference materials offer insight into how true (that is, accurate) analytical results are, how repeat measurements show how reproducible (that is, precise) analytical results are, and how blank samples can identify and track potential sources of contamination.

\subsubsection{Reference Materials}

Reference materials (RMs) are samples that have been tested in many different laboratories or by several different analytical techniques and given a certified value with an uncertainty. When used appropriately, RMs reflect the accuracy of the analytical results. By inserting RMs in sample sets, the results from RMs can be compared to the expected values so the researcher can assess the accuracy of the analytical values for the unknown samples. To be effective, however, appropriate RMs must be used. RMs chosen should be of comparable composition and also of comparable concentrations and similar matrices. For example, if samples are freshwater lacustrine waters, seawater RMs are not likely to provide meaningful information. Likewise, if samples are anticipated to contain 50 parts per billion (ppb) Hg, a RM with 5,000 ppb Hg would be inappropriate. In some cases, RMs are provided in a different physical state than the samples in the set and are therefore clearly known to be RMs to the analyst. For example, there are no RMs for particulate matter collected on filters, so solid RMs in jars are provided for analysis with the filters. When possible, however, blind standards may provide a better check of analytical accuracy. Blind standards are RMs that are given a sample sequential identification so the analyst is unaware that it is a certified RM, thus providing the scientist with an independent check on the data. Submittal of blind RMs is not an attempt to trick the analyst but a step to ensure that all samples (unknowns and RMs) are treated the same. This practice eliminates the subconscious "handling with kid gloves” of RMs. A list of sources for many RMs can be found in appendix 2.

It may also be important to develop an in-house standard (for example, site/study reference sample) that can be submitted with every sample set over a period of years or decades. To this end, Eastern Energy Resources Science Center (EERSC) scientists have collected approximately 30 pounds of Appalachian Basin feed coal from a powerplant in Kentucky. The homogenized powder is not a calibrated standard but can be included with other coal sample submissions, essentially acting as both a long-term duplicate and an in-house standard. Tracking value changes in this one sample over a long period will offer information on long-term instrument drift and reliability, true analytical uncertainties, cross checking between laboratories (if more than one laboratory is used or a need to switch laboratories arises), and quality assurance of other data. If no in-house standard is available, a previously measured sample can be resubmitted. 


\subsubsection{Duplicate and Replicate Samples}

Several types of duplicates are available, each of which offers useful information, but all are measures of different types of precision. Total analytical duplicates are two samples collected in tandem from the same source at the same time and treated individually during preparation and analysis. Results for total analytical duplicate samples do not need to be identical but should behave in some predictable manner. Even if the exact data reported for duplicates vary widely, if they fall on the same isochron or mixing line, it may simply be a case of natural variation or heterogeneity and not necessarily an analytical error (fig. 1).

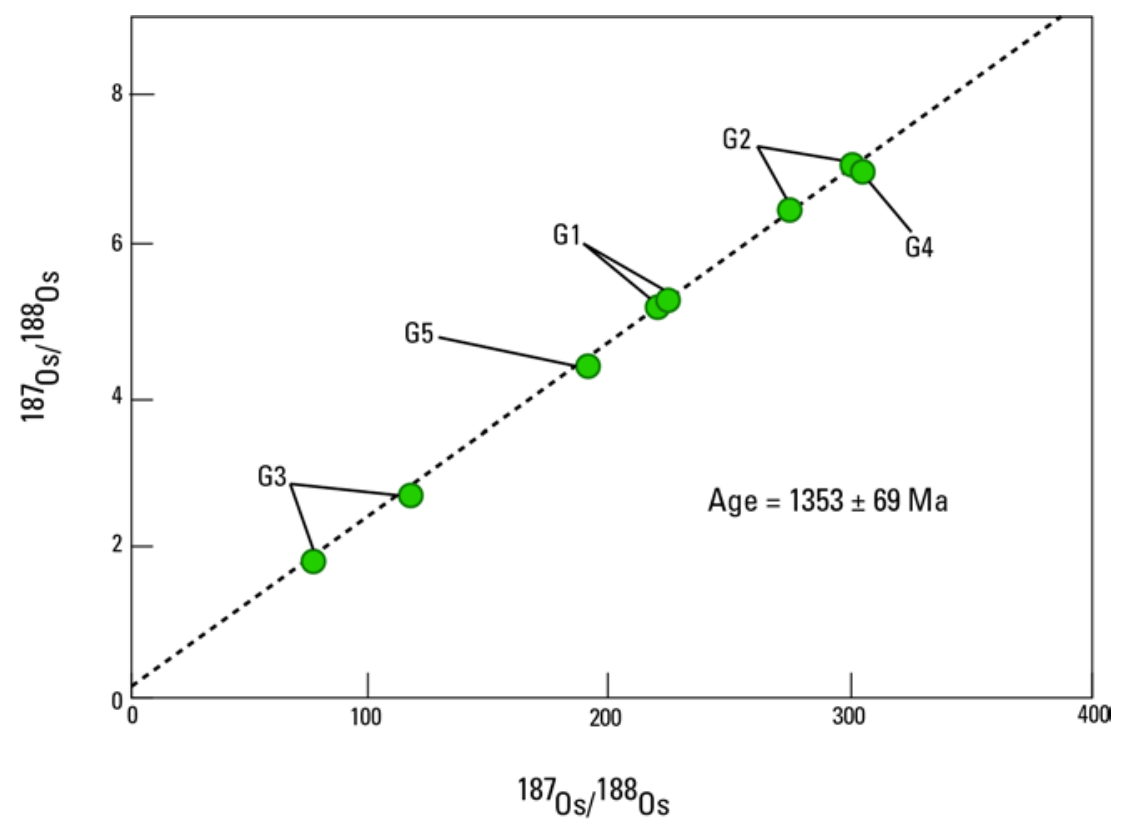

Figure 1. Data used to generate a rhenium (Re)-osmium (Os) isochron. Samples $\mathrm{G} 1, \mathrm{G} 2$, and $\mathrm{G} 3$ are total analytical duplicates. Although the samples do not reproduce well, they behave in a predictable manner (falling on the same isochron), suggesting sample heterogeneity rather than analytical error. Data from Geboy (2006); $\mathrm{Ma}=$ mega annum.

Replicates are aliquots of the same sample analyzed repeatedly on the instrument and are expected to reproduce results consistently. The standard deviation among replicate samples reflects the precision (or lack thereof) for that particular analysis and that particular sample. For example, if two aliquots of the same digestate are analyzed 10 samples apart from each other during analysis with widely different results, a large uncertainty will be associated with that sample that may affect what conclusions can be drawn from the data generated during that analysis. As with RMs, duplicate and replicate samples should be submitted as blind samples when possible.

\subsubsection{Blank Samples}

Blank samples are another vital component of any comprehensive dataset. Blanks offer insight on potential sample contamination sources and the appropriateness of methods and can be used to correct quantitative and isotopic data. Like duplicates, there are several forms of blanks. Blanks that are collected in the field have already been discussed, but there are also laboratory blanks. Total analytical blanks are processed side-by-side with samples but without the sampled material (or use a very clean 
substitute for the sample, such as $18.2 \mathrm{M} \Omega$-cm water). Total analytical blanks are useful in that they reveal background levels and possible contamination for the entire laboratory process. If total analytical blanks are sufficiently below the concentrations measured in samples, the technique is a good fit for the study. If they reveal a significant contribution to one or more component(s) being analyzed, then troubleshooting may be necessary.

Troubleshooting requires comparison and investigation into reagent blanks and labware blanks. By analyzing reagents and labware used in the preparation work, the scientist can determine the source of the contamination and adjust the method or clean/replace the contaminating step accordingly. Once total analytical blanks are at sufficiently low levels, they are used to correct concentrations and, if applicable, isotopic ratios in the unknown samples. Blank samples are effective only if they are collected in the same manner as regular samples. Like RMs, scientists may subconsciously use more care with blank samples to minimize their contribution. If blanks are prepared more cautiously than samples, they are potentially undervaluing actual external contributions.

\subsubsection{Suggested Inclusion of QA/QC Samples to Accompany a Batch of Samples Submitted to an Analytical Laboratory}

In order to provide confidence in data, Reimann and others (2008) suggest that all actual samples should be randomized to spread out any temporal systematic biases such as instrument drift. For example, consider a job that includes fly ash and bed or bottom ash samples. All of the fly ash samples are numbered sequentially, followed by all of the bed or bottom ash samples. If there is a drift in the instrument, which causes a slow decrease in signal strength over time, the fly ash samples (which were all analyzed early in the run) will be artificially higher than the bed ash samples (which were analyzed toward the end of the run). Randomizing samples helps to disperse this bias across all sample types. In addition to randomizing samples, Reimann and others (2008) suggest that sample sets include

- Field duplicates (5-10 percent of samples)

- Certified RMs (at least 1 percent of samples)

- In-house internal laboratory standard (10-20 percent of samples)

- Analytical duplicates (10-20 percent of samples)

These numbers cannot be met for all sample sets. In the case of coal cores or soil water, duplicates can be difficult, if not impossible, to obtain. The above percentages are simply suggestions and represent an ideal case. In instances where analytical techiques are particularly difficult or prone to contamination, an even higher frequency of QA/QC samples may be necessary.

\section{Post Laboratory Analysis}

Once results are returned from the laboratory, it is the responsibility of the scientist to review the results and check for discrepancies and QA/QC issues as soon as possible. If errors or questionable data are found, reporting them promptly may allow the analyst to rerun samples before they are disposed of. The laboratory report should routinely include internal QA/QC runs, such as calibration and standards, which bracket submitted samples. If these data are not included, they should be requested immediately. Determining percentage recovery of blind standards and comparison of duplicates will also offer insight into the quality of the data. If recovery is good (90-110 percent) for most components but poor for one or two, then data for those one or two components may not be reliable, and their inclusion in the results should be reconsidered. Results, even if tenuous, should never be eliminated or destroyed. Rather, data can be excluded from interpretation with an explanation of why they are excluded. If a long-term internal RM is being used, its values should be plotted every time it is analyzed and compared to 
previous analyses. This long-term plot can be especially useful if there has been a change in analyst, instrument, or laboratory used.

\subsection{Using Field Blanks to Determine the Method Detection Limit}

Several different thresholds can be used to evaluate the minimal meaningful quantitative value of data, such as the instrument detection limit (IDL) and the method detection limit (MDL). Analytical instruments will produce a small signal (that is, background noise) even when no sample has been injected into the machine. The IDL typically is defined as three times that of background. These detection limits are the ones reported by manufacturers and are often highly optimistic, meaning they are the best limits ever achieved under ideal running conditions. In addition to instrument noise, most samples have other sources of uncertainty associated with collection and sample preparation; therefore, the IDL is often much lower than the true detection limit of an entire method. A more realistic way of determining the detection limit for a sample is to calculate the MDL, which is defined as the minimum concentration of a component in a particular sample that can be measured and reported with 99 percent confidence that the concentration of the analyte is above zero. When results are returned from a laboratory, detection or reporting limits are included for each analysis. These limits are typically a form of the MDL, but it is important to confirm this with the analyst. National Energy Technology Laboratory (NETL) in Pittsburgh, Pennsylvania, for example, uses three times the standard deviation of 10 replicates of blank solution (Kristen Carlisle, National Energy Technology Laboratory, written commun., February 17, 2011). The USGS Minerals Resources Program laboratories in Denver calculate detection limits as three times the standard deviation of eight replicates of blanks that have gone through the same physical and chemical laboratory procedures as the samples (Paul Lamothe, USGS, written commun., February 17, 2011). These detection limits do not, however, take into account contribution from outside sources before they arrive in the laboratory. The best practice, therefore, is to calculate the MDL as three times the standard deviation of all the collected and analyzed field blanks or total analytical blanks, because the method begins long before samples are handled by the analyst.

Consider five field blanks that were collected during a recent field campaign. The main constituent of interest is lead $(\mathrm{Pb})$. The results for the blanks returned from the laboratory are 0.2883 , $0.1146,0.0761,0.1298$, and 0.0986 microgram $(\mu \mathrm{g})$. The standard deviation of these five blank values is $0.0843 \mu \mathrm{g}$. These blanks have recorded any contaminant contribution from the entire process of collecting, preparing, and analyzing the samples, meaning they represent the minimum amount of $\mathrm{Pb}$ that would be found in a sample that does not actually come from the sample itself; the blanks act as the chemical equivalent of a tare on a balance. Also, remember that (assuming normality) 99 percent of all values will fall within three times the standard deviation; therefore, a MDL of $0.0843 \mu \mathrm{g}$ times three is equal to $0.2529 \mu \mathrm{g}$ and is the minimum amount of actual contribution from the Pb sample that can be measured and reported with 99 percent confidence. MDLs are unique to each method and analyte and must be calculated by the researcher once results are obtained from the laboratory.

\subsection{Examples of the Importance of Scrutinizing Data with Respect to QA/QC}

Case Study 1 - Samples of atmospheric particulate matter were collected in Woods Hole, Massachusetts, during the last week of February 2008. The aerosols were collected over approximately 12-hour periods in fractions of less than $2.5 \mu \mathrm{m}$ (PM2.5) and total suspended particulates (TSP). Although the samples were collected continuously, the scientist sent the filters in two different batches several months apart to the same laboratory to be analyzed for trace elements. The results for three elements are shown in figure 2 with the vertical dash line separating the two different jobs. 
A severe drop in concentrations (in most cases, a full order of magnitude) of all elements occurred in both fractions between the two subsets. It is unlikely that an environmental change occurred, coinciding with where the scientist arbitrarily chose to divide the time series. Rather, an analytical error is more reasonable. After contacting the analyst, there was no obvious explanation for the discrepancy. Whether because of an undocumented dilution, an instrumental error, or an incomplete digestion, the QA/QC on these data are suspect, and therefore, they were not used in developing conclusions in the study.
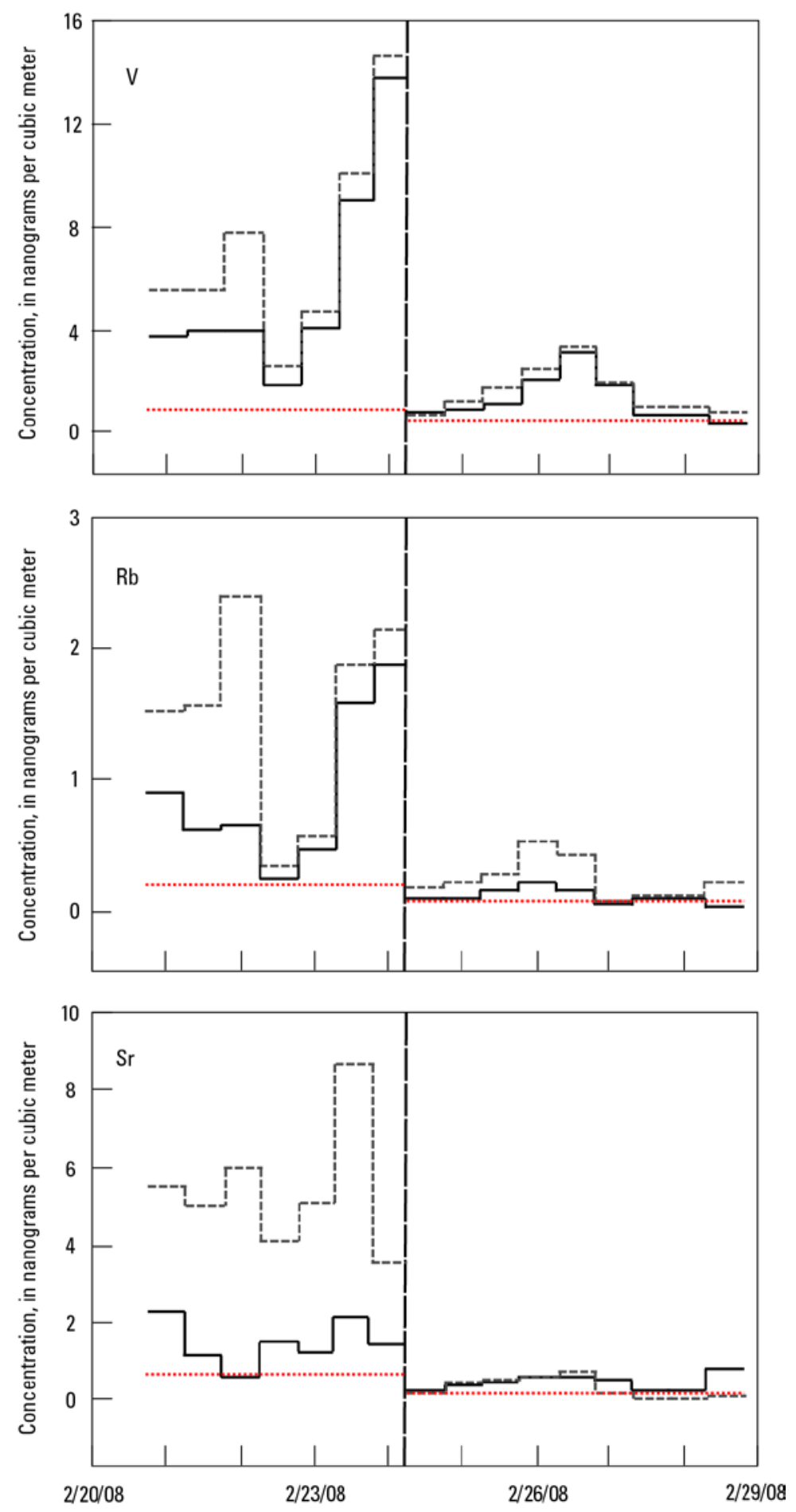

Figure 2. Vanadium, rubidium, and strontium concentration data in atmospheric particulate matter collected from Woods Hole, Massachusetts, in February 2008. The dashed line is total suspended particulate data, and the solid line represents fine particulate matter data. The red dotted line indicates the method detection limit. Note the abrupt decrease in concentrations between the first and second subset. Unpublished data from Mark Engle. 
Case Study 2 - Leachates from coal combustion products were analyzed for trace elements using both ICP-AES and ICP-MS. This test was designed to compare results of the two analytical methods for elements that were successfully analyzed by both instruments. The leachate samples were prepared using two different separation standards: toxicity characteristic leaching procedure (TCLP; U.S. Environmental Protection Agency, 1992) and synthetic groundwater leaching procedure (SGLP; as in Hassett, 1994). Ideally, both analytical procedures should yield similar results across most elements for each of the leaching standards. That is, copper concentrations from a TCLP leachate measured with ICP-AES should be similar to copper concentrations from a TCLP leachate measured with ICP-MS. Although ICP-AES more accurately measures certain elements than ICP-MS, and vice-versa, there is not a resolvable difference for many elements; therefore, if plotted against each other, ICP-AES data and ICP-MS data should have an approximately linear relation with a slope equal to one. The results received from the laboratory are plotted in figure 3.

The data do not show any sort of predictable relation. Initially, this scatter suggests that either one of the standard leaching protocols or one or both of the analytical techniques encountered significant problems. After looking more critically at the data, the scientist noticed that if the laboratory IDs for a run of samples were transposed (that is, SGLP and TCLP samples were switched), the data would plot in a more agreeable manner, as seen in figure 4. Following a consultation with the analyst, who checked his laboratory notebook, a data transfer error was found. It appears both standards and analytical methods produce similar results with the notable exception of arsenic (and possibly nickel, cobalt, and selenium). In this case, a careful review of the data saved the time and money of unnecessary troubleshooting.

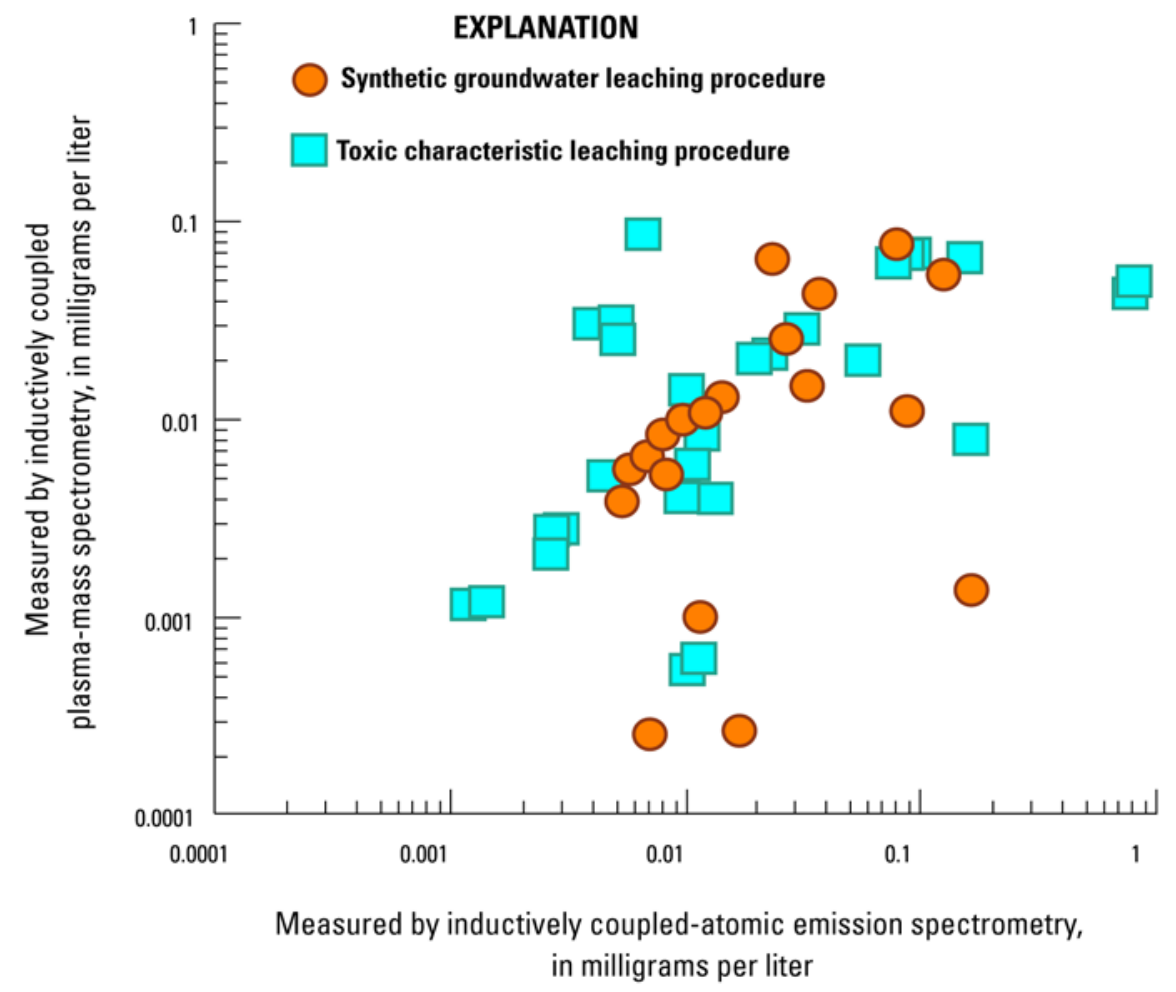

Figure 3. Leachate data from coal combustion products by two different analytical procedures, which do not display a 1:1 linear relation. Data from Jones and others (2010). 


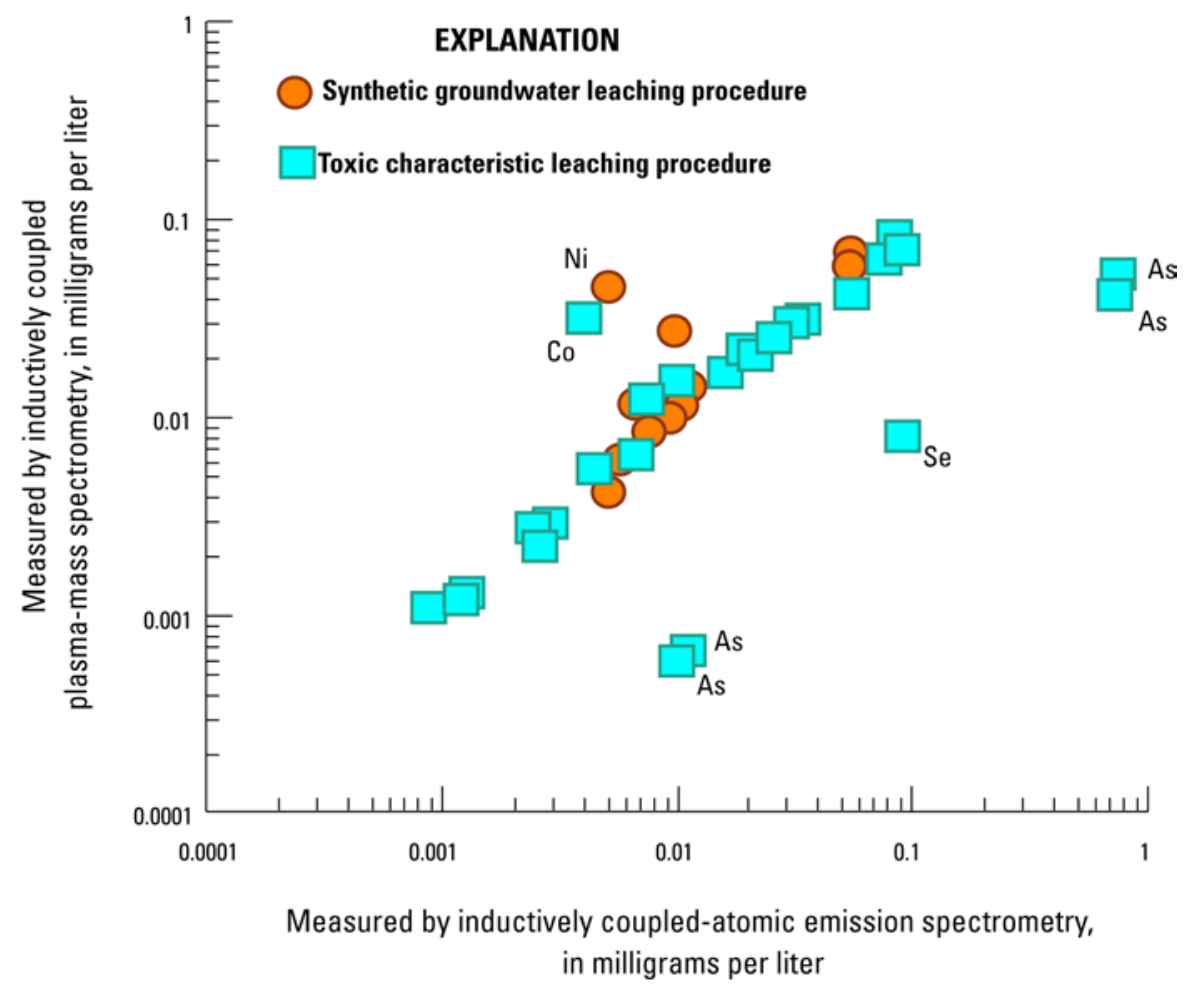

Figure 4. Leachate data from coal combustion products by two different analytical procedures following a transposition of laboratory identifications. With the notable exception of arsenic (As), the data now approximately reflect the expected 1:1 linear relation. Data from Jones and others (2010); $\mathrm{Ni}=$ nickel; $\mathrm{Co}=$ cobalt; $\mathrm{Se}=$ selenium.

\subsection{Dealing with Censored Data}

Once the data have been verified by the scientist, they need to be put into context and interpreted. This process sometimes requires statistical treatment of the data, which can be complicated by the presence of censored data. Often, some fraction of the analytical results for a given constituent will fall below the IDL or MDL. Data that are below detection are referred to as nondetects or "censored data" and are reported by the analyst as not detected or $<\mathrm{X}$, where $\mathrm{X}$ is the appropriate detection limit. Handling these data correctly is vital in assuring the quality of any conclusions drawn from the data.

Traditionally, scientists have substituted a nominal value, such as zero, one-half, or two-thirds of the detection limit, for the censored values and then proceeded with interpreting the data and calculating summary statistics. Over the last decade, however, statisticians and chemometricians have commented on this substitution method; for example, “...substituting values for nondetects should be used rarely, and should generally be considered unacceptable in scientific research. There are better ways” (Helsel, 2006, p. 2434). The argument is made that substituting censored values is a misrepresentation that amounts to falsifying data and can lead to serious errors in any statistical information derived from that dataset. Rather, it has been suggested that censored values can be treated statistically by using several methods, including maximum-likelihood estimates (MLE), Kaplan-Meier (K-M) survival curves, and regression on order statistics (ROS). The following section briefly explains how these methods can be applied to estimate summary statistics from datasets that contain nondetects. In addition, methods have been developed for regression/correlation and statistical comparison among groups but are not discussed here (Helsel, 2005). 
To determine which method is most suitable for a particular dataset, the important things to consider are the amount of data available, the percentage of the sample set that is censored, and the distribution of those data reported above detection limits. The K-M method is a nonparametric analysis based on the probability of a sample "surviving" detection and will accommodate datasets regardless of their distributions (that is, Gaussian, lognormal, bimodal, random, and so forth). The limitation of the KM method is that it cannot determine the median if one-half the data (or more) are censored. Likewise, it cannot determine the first quartile if more than 25 percent of the data are censored, and so on. In addition, if there is only a single detection limit for the component being analyzed and none of the samples exhibit a detected concentration below that detection limit, the K-M method is essentially equivalent to a substitution method. In these situations it may be more prudent to employ either MLE or ROS methods. Both of these methods, however, also come with restrictions and assumptions. MLE is applicable only to datasets that closely follow a given distribution (for example, normal, log normal, and so on) as it fits a curve to the detected values and extrapolates to points below the detection limit. Summary statistics are then generated for the curve that fits that distribution. This method is most effective with larger sample sizes ( $\mathrm{n}>50$ ). The ROS method is similar but applies the distribution only to the fraction of censored values. Data points above the detection limit are used in combination with that "below detection" distribution to generate summary statistics. The ROS method works well with smaller datasets $(n<50)$. Helsel (2005) provides a full review of these methods. If more than 80 percent of the data are censored or if the dataset is small or both, then all of these statistical methods for censored data may yield tenuous results that may not provide meaningful statistics. Then again, if more than 80 percent of the data are censored, it may also mean the analytical method is inappropriate. In addition, Olea and others (2008) have suggested applying bootstrapping methods to estimate summary statistics for datasets that are treated using compositional data methods. Several statistical packages, including Minitab, NCSS, S-PLUS, and the NADA library for R, can execute one or more of these methods.

As an example of how the substitution method can alter true statistics, consider sodium concentration data collected from atmospheric total suspended particulate matter in Woods Hole, Mass. (table 1). In actuality, none of the data were reported below the MDL, so the "true" summary statistics can be determined: median of $590 \mathrm{ng} \mathrm{m}^{-3}$; mean of $658 \mathrm{ng} \mathrm{m}^{-3}$; and a standard deviation of $321 \mathrm{ng} \mathrm{m}^{-3}$. As an exercise, the detection limit is arbitrarily set to different concentrations to censor different percentages of the data. In each of these cases, the censored data are replaced with one-half the arbitrary detection limit, and statistics were determined using the substitution (SUBS), K-M, ROS, and MLE methods (table 2).

The summary statistics generated from the substitution method agree with the actual summary statistics when only 17 percent of the data are censored, but as the detection limit rises (and the percentage of data falling below that detection limit increases), substitution produces drastic negatively biased results. The calculated median deviates from the actual median by almost 25 percent and 40 percent when 50 percent and 57 percent of the data are censored, respectively, while the ROS method deviates by only $<1$ percent and 3 percent, respectively. These data are lognormal (they are linear on a log-probability plot), so either the MLE or ROS could be applied. Again, the ROS method is preferred with smaller datasets, and these results support that preference. The ROS method provides fairly consistent median, mean, and standard deviation results even when 70 percent of the data are censored. Table 2 also illustrates that the K-M method cannot determine a median (that is, a second quartile) when 50 percent or more of the data fall below detection. As pointed out above, in this particular usage where there is a single detection limit and no samples with detected values below that limit, the K-M method behaves as a variation of the substitution method. 
Table 1. Sodium ( $\mathrm{Na}$ ) concentration data for atmospheric total suspended particulate matter from Woods Hole, Massachusetts, with summary statistics.

[ng/m ${ }^{3}$, nanograms per cubic meter]

\begin{tabular}{lclr}
\hline Sample ID & $\mathrm{Na}\left(\mathrm{ng} / \mathrm{m}^{3}\right)$ & Sample ID & $\mathrm{Na}\left(\mathrm{ng} / \mathrm{m}^{3}\right)$ \\
\hline 08-MA-06TF & 876 & 08-MA-100TF & 1,440 \\
08-MA-09TF & 72.6 & 08-MA-03TF & 495 \\
08-MA-10TF & 1,400 & 08-MA-60TF & 245 \\
08-MA-12TF & 277 & 08-MA-62TF & 169 \\
08-MA-13TF & 282 & 08-MA-63TF & 438 \\
08-MA-14TF & 713 & 08-MA-64TF & 555 \\
08-MA-15TF & 852 & 08-MA-65TF & 887 \\
08-MA-22TF & 747 & 08-MA-66TF & 576 \\
08-MA-24TF & 711 & 08-MA-68TF & 515 \\
08-MA-26TF & 841 & 08-MA-72TF & 995 \\
08-MA-30TF & 644 & 08-MA-07TF & 475 \\
08-MA-34TF & 1,110 & 08-MA-82TF & 790 \\
072908-1 & 574 & 08-MA-83TF & 527 \\
08-MA-95TF & 603 & 08-MA-85TF & 569 \\
08-MA-98TF & 475 & 08-MA-92TF & 890 \\
\hline Median & $\mathbf{5 9 0}$ & & \\
Mean & $\mathbf{6 5 8}$ & & \\
Standard Deviation & $\mathbf{3 2 1}$ & & \\
\hline
\end{tabular}


Table 2. Comparison of summary statistics for the dataset when arbitrary detection limits are set and four different nondetect statistical methods are employed.

[K-M, Kaplan-Meier; ROS, regression on order statistics; MLE, maximum likelihood estimates; SUBS, substitution method; NA, not applicable]

\begin{tabular}{|c|c|c|c|c|c|c|}
\hline Detection limit & $\begin{array}{l}\text { Percent } \\
\text { censored }\end{array}$ & & K-M & ROS & MLE & SUBS \\
\hline \multirow[t]{3}{*}{300} & 17 & Median & 576 & 590 & 518 & 590 \\
\hline & & Mean & 696 & 678 & 695 & 648 \\
\hline & & Standard Deviation & 273 & 293 & 623 & 334 \\
\hline \multirow[t]{3}{*}{500} & 30 & Median & 576 & 590 & 480 & 590 \\
\hline & & Mean & 715 & 672 & 651 & 636 \\
\hline & & Standard Deviation & 258 & 298 & 595 & 337 \\
\hline \multirow[t]{3}{*}{600} & 50 & Median & NA & 595 & 350 & 451 \\
\hline & & Mean & 751 & 668 & 587 & 600 \\
\hline & & Standard Deviation & 233 & 301 & 790 & 351 \\
\hline \multirow[t]{3}{*}{710} & 57 & Median & NA & 609 & 337 & 355 \\
\hline & & Mean & 811 & 674 & 556 & 609 \\
\hline & & Standard Deviation & 198 & 296 & 730 & 355 \\
\hline \multirow[t]{3}{*}{800} & 70 & Median & NA & 584 & 233 & 400 \\
\hline & & Mean & 899 & 652 & 469 & 590 \\
\hline & & Standard Deviation & 159 & 306 & 821 & 320 \\
\hline
\end{tabular}

\section{Closing Remarks}

While it may be easy or convenient to trust analytical data, it is not always prudent. Some data are clearly erroneous and, although not used, represent a waste of effort and funding. Even more problematic, however, are incorrect data that are inadvertently published. Following proper QA/QC and sampling methods from the inception of a project through its completion can greatly increase confidence that the data are reliable. As geochemical tools become more available, careful QA/QC procedures become even more important. Analytical techniques are becoming more refined, and detection limits are being reduced. With this refinement, even minor contamination can cause differences in data that can drastically change interpretations and conclusions. Rapidly improving geochemical techniques require that more time be spent reviewing recent literature prior to the proposal of a study. Published method sections that are only a few years old may have been subsequently improved upon and, therefore, rendered obsolete.

Keeping lines of communication open with the laboratory is beneficial to all parties. Laboratory managers and analysts may have first-hand experience with techniques that have or have not been successful in the past. Also, the more information that is given to the laboratory, the better the laboratory can tailor the analyses to help achieve the goals of the study. It also helps if a rapport exists among all people involved with the study so that, if problems do arise, they can be handled in a coordinated, professional, and timely manner. 
Developing a study, collecting and processing samples, analyzing for key elements and compounds, and reviewing results all require appropriate QA/QC protocols. QA/QC work may be iterative and may not end even when the study ends. While this primer is not intended to be a comprehensive guide to QA/QC within the USGS Energy Resources Program, it may offer insight into good QA/QC practices in general. Ultimately, the QA/QC work may provide information as important as the original data themselves. At the very least, closely following QA/QC guidelines will lead to the reliable data that are expected by USGS stakeholders and the public.

\section{References}

Brocks, J.J., Buick, Roger, Logan, G.A., and Summons, R.E., 2003, Composition and syngeneity of molecular fossils from the 2.78 to 2.45 billion-year-old Mount Bruce Supergroup, Pilbara Craton, Western Australia: Geochimica et Cosmochimica Acta, v. 67, issue 22, p. 4289-4319.

Fitton, Godfrey, 1997, X-ray fluorescence spectrometry, in Gill, Robin, ed., Modern analytical geochemistry: Essex, U.K., Addison Wesley Longman, p. 87-115.

Geboy, Nicholas, 2006, Rhenium-osmium age determinations of glaciogenic shales form the Mesoproterozoic Vazante Formation, Brazil: University of Maryland, Master's Thesis, 99 p. Gill, Robin, ed., 1997, Modern analytical geochemistry: Essex, U.K., Addison Wesley Longman, 327 p.

Hassett, D.J., 1994, Scientifically valid leaching of coal conversion solid residues to predict environmental impact: Fuel Processing Technology, v. 39, issue 1-3, p. 445-449.

Helsel, D.R., 2005, Nondetects and data analysis-Statistics for censored environmental data: New York, Wiley, 250 p.

Helsel, D.R., 2006, Fabricating data-How substituting values for nondetects can ruin results, and what can be done about it: Chemosphere, v. 65, no. 11, p. 2434-2439.

Helsel, D.R., and Hirsch, R.M., 2002, Methods for data below the reporting limit, Chapter 13, in Statistical methods in water resources, U.S. Geological Survey Techniques of Water-Resources Investigations, book 4, chap. A3, p. 357-376.

Jarvis, K.E., 1997, Inductively coupled plasma-mass spectrometry (ICP-MS), in Gill, Robin, ed., Modern analytical geochemistry: Essex, U.K., Addison Wesley Longman, p. 171-187.

Jones, K.B., Ruppert, L.F., Swanson, S.M., and Affolter, R.H., 2010, Trends in elemental leaching from coal combustion products from two coal-fired power plants: Geological Society of America Abstracts with Programs, v. 42, no. 5, p. 610.

Luppens, J.A., Wilson, S.E., and Stanton, R.W., eds., 1992, Manual on drilling, sampling, and analysis of coal: Philadelphia, Pa., American Society for Testing and Materials, 61 p.

Olea, R.A., 2008, Inference of distributional parameters of compositional samples containing nondetects: Proceedings from the 3d Compositional Data Analysis Workshop CoDaWork '08, Girona, Spain, May 27-30, 2008, 20 p.

Parry, S.J., 1997, Neutron activation analysis, in Gill, Robin, ed., Modern analytical geochemistry:

Essex, U.K., Addison Wesley Longman, p. 116-134.

Ramsey, M.H., 1997, Sampling and sample preparation, in Gill, Robin, ed., Modern analytical geochemistry: Essex, U.K., Addison Wesley Longman, 327 p.

Reimann, C., Filzmoser, P., Garrett, R., and Dutter, R., 2008, Statistical data analysis explained: West Sussex, U.K., John Wiley \& Sons, Ltd., 343 p.

Rowland, A.P., 1997, Atomic absorption spectrometry and other solution methods, in Gill, Robin, ed., Modern analytical geochemistry: Essex, U.K., Addison Wesley Longman, p. 67-86.

Skoog, D.A., Holler, F.J., and Crouch, S.R., 2007, Principles of instrumental analysis (6th ed.): Belmont, Calif., Thomson Brooks/Cole, 1039 p. 
U.S. Army Corps of Engineers, 2005, Environmental quality-Guidance for evaluating performancebased chemical data: Pub \#EM 200-1-10.

U.S. Environmental Protection Agency, 1999, Compendium of methods for the determination of inorganic compounds in ambient air: EPA/625/R-96/010a, accessed July 1, 2011, at http://www.epa.gov/nrmrl/pubs/625r96010/iocompen.pdf.

U.S. Environmental Protection Agency, 1992, EPA Test Method 1311-TCLP, Toxicity characteristic leaching procedure, accessed July 1, 2011, at http://www.ehso.com/cssepa/TCLP_from\%20EHSOcom_Method_1311.pdf.

U.S. Environmental Protection Agency, 2007, Method 7000B-Flame atomic absorption spectrophotometry, accessed July 1, 2011, at http://www.epa.gov/osw/hazard/testmethods/sw846/pdfs/7000b.pdf.

U.S. Geological Survey, 2006, Collection of water samples: U.S. Geological Survey Techniques of Water-Resources Investigations, book 9, chap. A4, accessed July 1, 2011, at http://pubs.water.usgs.gov/twri9A4/.

Walsh, J.N., 1997, Inductively coupled plasma-atomic emission spectrometry (ICP-AES), in Gill, Robin, ed., Modern analytical geochemistry: Essex, U.K., Addison Wesley Longman, p. 41-66. 


\section{Appendix 1}

\section{Detection Limits for Various Analytical Techniques}

The figures in this appendix present detection limits for various analytical techniques. For the convenience of the user, each analytical technique has its own periodic table; shading is used to identify the order of magnitude to which that technique can measure each element. The detection limits are labeled in parts per million (ppm) and parts per billion (ppb), which are equivalent to milligrams per liter $(\mathrm{mg} / \mathrm{L})$ and micrograms per liter $(\mu \mathrm{g} / \mathrm{L})$, repectively, for samples in solution and micrograms per gram $(\mu \mathrm{g} / \mathrm{g})$ and nanograms per gram $(\mathrm{ng} / \mathrm{g})$, respectively, for solid samples.

\section{Detection Limits, ICP-AES}

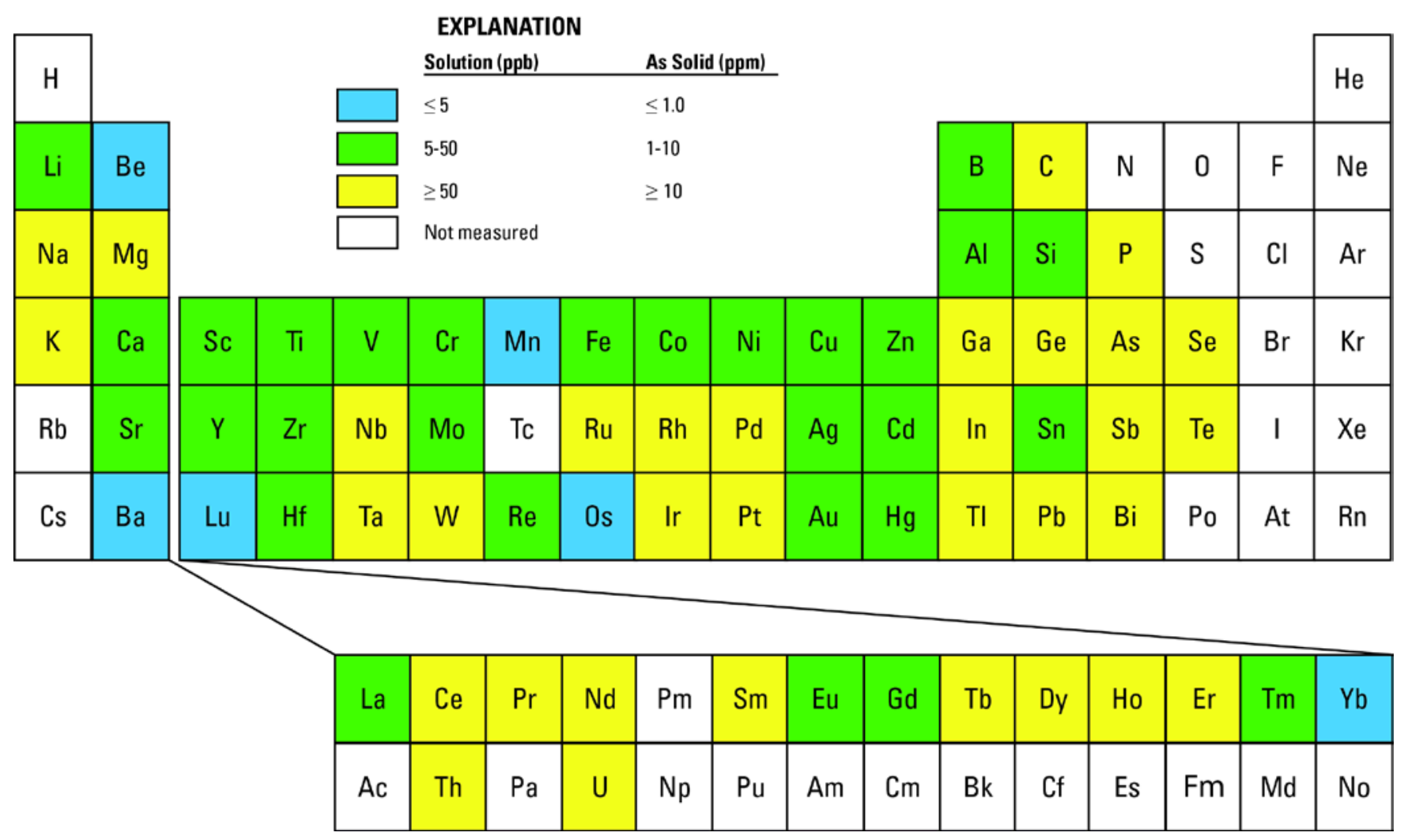

Figure A1. Instrument detection limits for inductively coupled plasma-atomic emission spectrometry (ICP-AES). From Walsh, 1997; ppm, parts per million; ppb, parts per billion. 
Detection Limits, ICP-MS

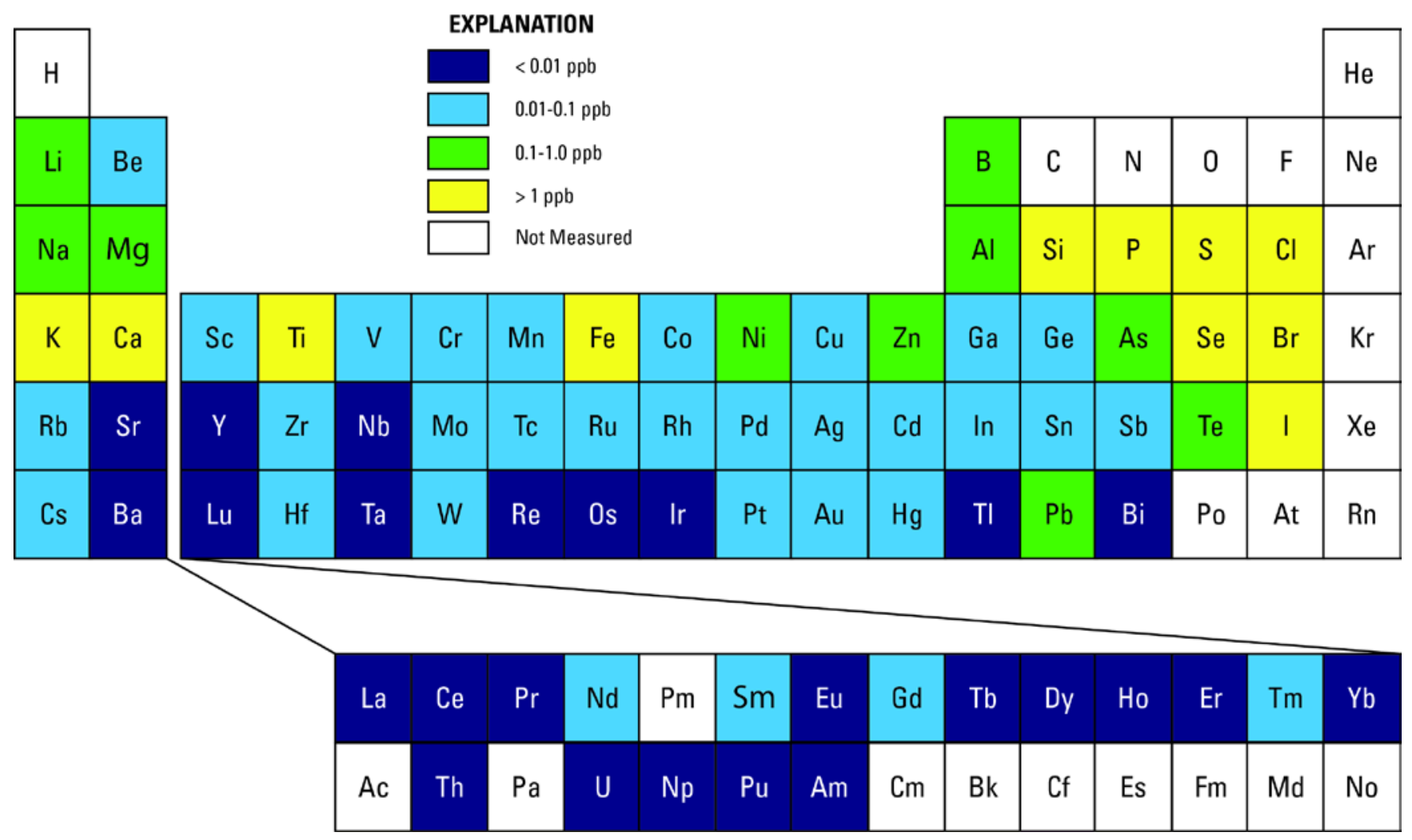

Figure A2. Instrument detection limits for inductively coupled plasma-mass spectrometry (ICP-MS). From Jarvis, 1997; ppm, parts per million. 
Detection Limits, NAA

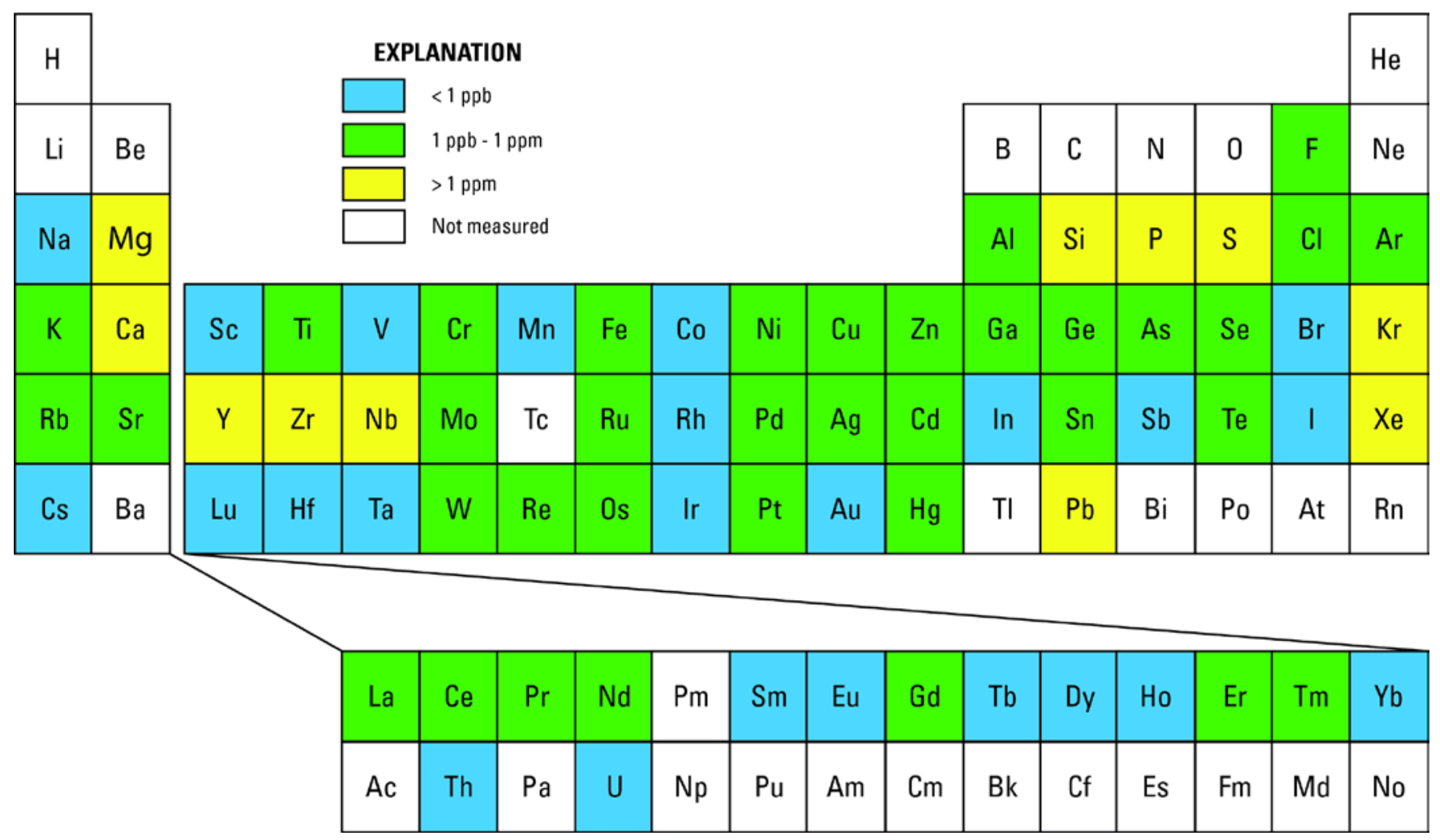

Figure A3. Instrument detection limits for neutron activation analysis (NAA). From Parry, 1997; ppm, parts per million; ppb, parts per billion. 
Detection Limits, XRF

\begin{tabular}{|c|c|c|c|c|c|c|c|c|c|c|c|c|c|c|c|c|c|}
\hline $\mathrm{H}$ & \multicolumn{16}{|c|}{ EXPLANATION } & \multirow{3}{*}{$\begin{array}{l}\mathrm{He} \\
\mathrm{Ne}\end{array}$} \\
\hline & & & & & & \multicolumn{11}{|c|}{$0.1-5 \mathrm{ppm}$} & \\
\hline $\mathrm{Li}$ & $\mathrm{Be}$ & & & & & \multirow{2}{*}{\multicolumn{6}{|c|}{$\begin{array}{l}\text { 5-50 ppm } \\
50-500 \mathrm{ppm} \\
\text { Not measured }\end{array}$}} & B & C & $\mathrm{N}$ & 0 & $\mathrm{~F}$ & \\
\hline $\mathrm{Na}$ & $\mathrm{Mg}$ & & & & & & & & & & & $\mathrm{Al}$ & $\mathrm{Si}$ & $P$ & S & $\mathrm{Cl}$ & $\mathrm{Ar}$ \\
\hline K & $\mathrm{Ca}$ & Sc & $\mathrm{Ti}$ & V & $\mathrm{Cr}$ & $\mathrm{Mn}$ & $\mathrm{Fe}$ & Co & $\mathrm{Ni}$ & $\mathrm{Cu}$ & $\mathrm{Zn}$ & $\mathrm{Ga}$ & $\mathrm{Ge}$ & As & Se & $\mathrm{Br}$ & $\mathrm{Kr}$ \\
\hline $\mathrm{Rb}$ & $\mathrm{Sr}$ & Y & $\mathrm{Zr}$ & $\mathrm{Nb}$ & Mo & Tc & $\mathrm{Ru}$ & $\mathrm{Rh}$ & $\mathrm{Pd}$ & $\mathrm{Ag}$ & $\mathrm{Cd}$ & In & Sn & $\mathrm{Sb}$ & $\mathrm{Te}$ & I & $\mathrm{Xe}$ \\
\hline Cs & $\mathrm{Ba}$ & Lu & $\mathrm{Hf}$ & $\mathrm{Ta}$ & W & $\operatorname{Re}$ & Os & Ir & $\mathrm{Pt}$ & $\mathrm{Au}$ & $\mathrm{Hg}$ & TI & $\mathrm{Pb}$ & $\mathrm{Bi}$ & Po & At & $\mathrm{Rn}$ \\
\hline & & & & La & $\mathrm{Ce}$ & $\operatorname{Pr}$ & $\mathrm{Nd}$ & $\mathrm{Pm}$ & Sm & Eu & $\mathrm{Gd}$ & $\mathrm{Tb}$ & Dy & Ho & $\mathrm{Er}$ & $\mathrm{Tm}$ & $\mathrm{Yb}$ \\
\hline & & & & Ac & Th & $\mathrm{Pa}$ & U & $\mathrm{Np}$ & $\mathrm{Pu}$ & $\mathrm{Am}$ & $\mathrm{Cm}$ & $\mathrm{Bk}$ & $\mathrm{Cf}$ & Es & $\mathrm{Fm}$ & Md & No \\
\hline
\end{tabular}

Figure A4. Instrument detection limits for X-ray fluorescence spectrometry (XRF). From Fitton, 1997; ppm, parts per million. 


\section{Detection Limits, Flame AAS}

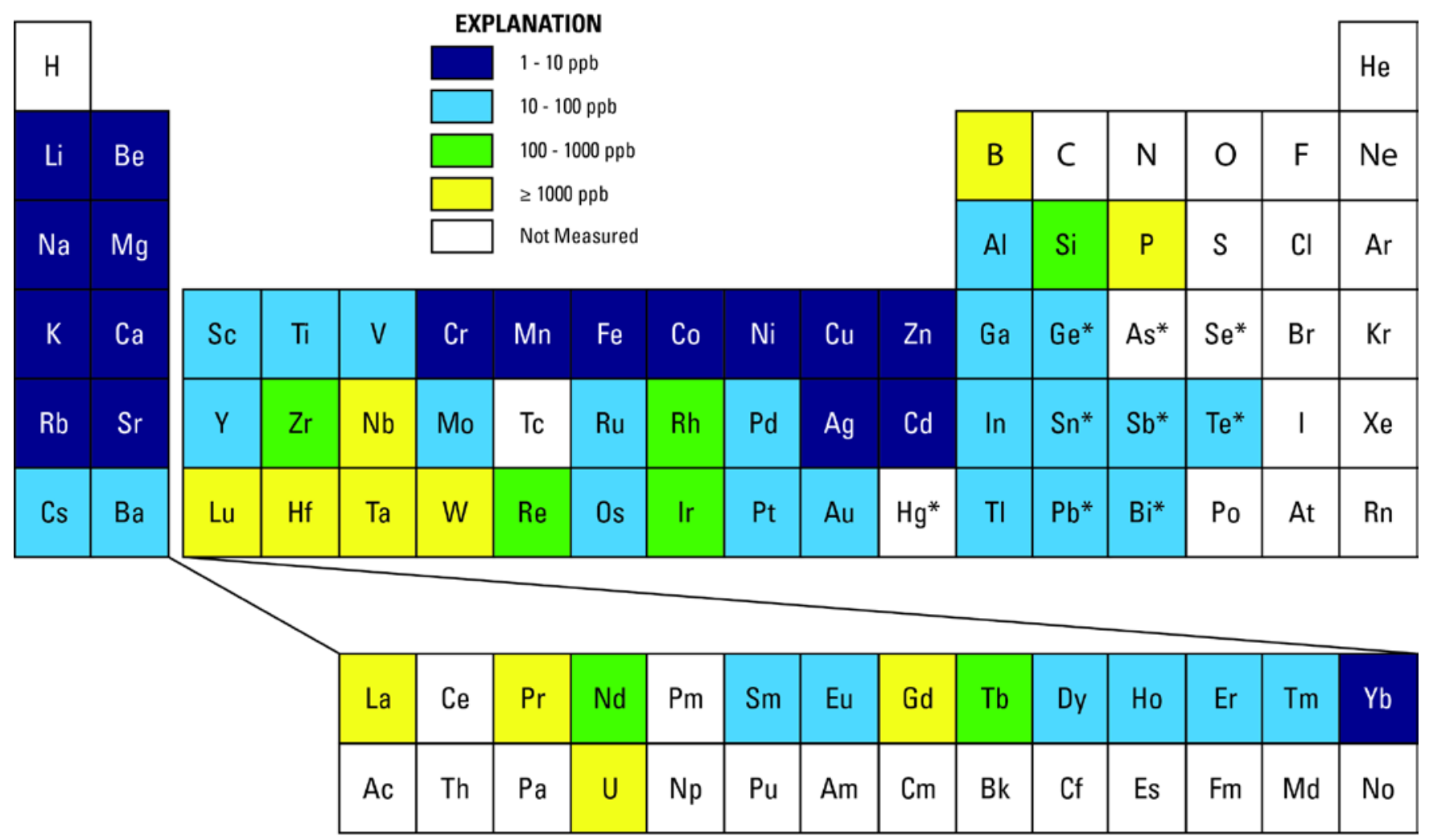

* Detection limit falls to 1 - 10 ppb with Cold-vapor \& hydride AAS

Figure A5. Instrument detection limits for atomic absorption spectrometry (AAS). From Rowland, 1997; ppb, parts per billion. 


\section{Appendix 2}

\section{Where to Obtain Reference Materials}

\begin{tabular}{ll}
\hline CompanylAgency & Type of Resource Material \\
\hline U.S. Geological Survey, Mineral Resources & \\
Nevada Bureau of Mines and Geology & Hard Rock Samples \\
National Institute of Standards and Technology & Precious Metals \\
European Commission, Institute for Reference Materials and & Ores and Sediments, Natural Gas, Water \\
Measurements & Water, Soil and Air Quality \\
U.S. Geological Survey, Water Resources & Aqueous Trace Elements, Major Ions, \\
Inorganic Ventures & Nutrients and Mercury \\
National Research Council of Canada & Inorganics \\
Coal Standards & Biological and Hydrological \\
Scott Specialty Gases & Coal and Coal Components \\
Koehler Instruments & Natural Gas and Methane \\
Quality Assurance Resources & Petroleum \\
\hline
\end{tabular}

